\title{
WestVirginiaUniversity
}

THE RESEARCH REPOSITORY @ WVU

Graduate Theses, Dissertations, and Problem Reports

2011

\section{The Performance of Asynchronous Cooperative Diversity with SIC Receivers}

Harish Palakurthi

West Virginia University

Follow this and additional works at: https://researchrepository.wvu.edu/etd

\section{Recommended Citation}

Palakurthi, Harish, "The Performance of Asynchronous Cooperative Diversity with SIC Receivers" (2011). Graduate Theses, Dissertations, and Problem Reports. 4762.

https://researchrepository.wvu.edu/etd/4762

This Thesis is protected by copyright and/or related rights. It has been brought to you by the The Research Repository @ WVU with permission from the rights-holder(s). You are free to use this Thesis in any way that is permitted by the copyright and related rights legislation that applies to your use. For other uses you must obtain permission from the rights-holder(s) directly, unless additional rights are indicated by a Creative Commons license in the record and/ or on the work itself. This Thesis has been accepted for inclusion in WVU Graduate Theses, Dissertations, and Problem Reports collection by an authorized administrator of The Research Repository @ WVU. For more information, please contact researchrepository@mail.wvu.edu. 


\title{
The Performance of Asynchronous Cooperative Diversity with SIC Receivers
}

\author{
by \\ Harish Palakurthi \\ Thesis submitted to the \\ College of Engineering and Mineral Resources \\ at West Virginia University \\ in partial fulfillment of the requirements \\ for the degree of \\ Master of Science \\ in \\ Electrical Engineering \\ Daryl Reynolds, Ph.D., Chair \\ Matthew C.Valenti, Ph.D. \\ Vinod Kulathumani, Ph.D.
}

Lane Department of Computer Science and Electrical Engineering

Morgantown, West Virginia

2011

Keywords: Cooperative Diversity, MIMO, Decode and Forward, Asynchronous, MMSE, SIC

Copyright 2011 Harish Palakurthi 


\begin{abstract}
The Performance of Asynchronous Cooperative Diversity with SIC Receivers

by

Harish Palakurthi
\end{abstract}

The multiple-input multiple-output (MIMO) antenna systems are well known for achieving the higher spectral efficiencies and improving the link reliability in wireless communications. But the use of multiple antennas in a cellular uplink is impractical due to the size constraints of the mobile device. The potential solution which was used is to exploit the user cooperation to create a virtual antenna array. In this paper we investigate the cooperative diversity in an asynchronous code-division multiple-access (CDMA) uplink by taking inter-user nonorthogonality(allocation of non-orthogonal channels to multiple users) into consideration.

Most the previous research work assumes the use of the inter-user orthogonality and the synchronism between the signals transmitted from different users. Both of these assumptions might bring in extra complexities in the network as they both will need the users to be accurately coordinated. If we consider the inter-user nonorthogonality and the asynchronous communication between transmitter and the receiver, we will need a way to combat the multiple access interference (MAI) and the inter-symbol interference (ISI). In this thesis try to eliminate those effects by using the MMSE detector and Successive Interference Cancellation (SIC) receivers. These techniques help will enhance total network performance and can exploit maximum diversity. We will be assuming a CDMA cellular uplink model with a multi-user decode-and-forward (DF) cooperative diversity protocol wherein each user first transmits its own message and other users overhear that message and can relay it to base station. So each user here acts as a relay and also has its own information to transmit. The channel spans a single spreading code, $s_{k}(t)$ for two orthogonal time-phases. In the first time phase every user broadcasts its own spread message and in the second time phase, the users in the decoding set relays the re-encoded and re-spreaded messages. The relays which doesn't belong in the decoding set, continue to transmit their own information. But throughout the paper we consider that all of the users are cooperating and hence the decoding relays transmit asynchronously over the subchannel. And the use of non-orthogonal spreading codes leads to non-orthogonality across the subchannels. 
To my family and all my friends who supported me 


\section{Acknowledgments}

I would like to express my deepest gratitude to my advisor, Dr. Daryl Reynolds for his guidance and support by which i am able to complete this thesis of mine. I am very thankful for his patience, motivation and knowledge which helped me in all of my research and writing this thesis. I could not have imagined a better advisor and mentor to further continue my studies.A special thanks to Dr. Matthew Valenti and Dr. Vinod Kulathumani for being on my committee and for their encouragement and insightful comments and questions.

I am also thankful to Kanchan Vardhe for taking time to help me initially when i started my research and guide me through my thesis. I would also like to thank Dr. Afzel Noore and Brian Powell for offering me the graduate teaching assistantship and letting me teach which has helped me out a great deal. I thank my colleagues Jyothi Boppana, Ricky Hussmann, Sharma Kollaparthi, Veeru Talreja and Praveen Konatham for all the stimulating discussions we had throughout my Masters. I also thank my friends Surya, Ganesh, Vamsi, Parupati, Srinu, Pavan, Dandu, Nambu, Anand, Chandana and Sai Bharath for all the help and support.

Last but not the least i would like to thank my parents Manjula and Damodar Palakurthi and my sister Harika for continuously supporting me throughout my life. Without their encouragement and blessings it would have been impossible for me to finish this work. 


\section{Contents}

Acknowledgments $\quad$ iv

List of Figures $\quad$ vii

Notation $\quad$ ix

1 Introduction $\quad 1$

1.1 Thesis Outline . . . . . . . . . . . . . . . . . . 3

2 Wireless Communication Systems Overview and Background 6

2.1 Representation of Signals in a Communication System . . . . . . . . . . . 6

2.2 The Wireless Channel . . . . . . . . . . . . . . . . . . . 7

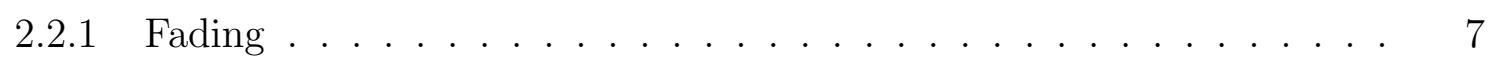

2.3 Diversity . . . . . . . . . . . . . . . . . . . . . . . . 9

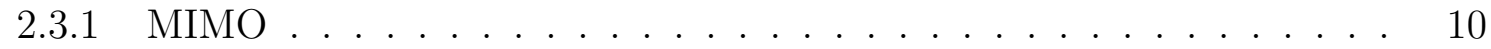

2.3.2 Cooperative Diversity . . . . . . . . . . . . . . . . . . 11

2.4 Multiple Access Techniques . . . . . . . . . . . . . . . . . . 13



3 Cooperative Asynchronous CDMA Uplink 20

3.1 Laneman's Distributed Space-Time Coded Cooperative Diversity Protocol . 21

3.1.1 System Model . . . . . . . . . . . . . . . . . . . . . . . 21

3.1.2 Outage Probability for Distributed STC . . . . . . . . . . . . . 23

3.2 Proposed Asynchronous CDMA uplink . . . . . . . . . . . . . . . . . 24

3.2 .1 System Model . . . . . . . . . . . . . . . . . . . . . . . . 24

3.2 .2 Decorrelator Detector . . . . . . . . . . . . . . 26

3.2 .3 MMSE Detector . . . . . . . . . . . . . . . . . 27

3.2 .4 SIC Receiver . . . . . . . . . . . . . . . . . . . . . . . . . . 29

4 Information-Outage Probability Analysis for MMSE and SIC Receivers ${ }^{1} 31$

4.1 Performance measures using MMSE receiver . . . . . . . . . . . . . . . 31

4.1.1 Underloaded CDMA Uplink . . . . . . . . . . . . . . . . . . 32

4.1.2 Fully-loaded CDMA Uplink . . . . . . . . . . . . . . . . 33

\footnotetext{
${ }^{1}$ The results shown in this chapter has been published in the proceedings of the 2011 Virginia Tech wireless symposium [1]
} 
4.1.3 Overloaded CDMA Uplink . . . . . . . . . . . . . . . . . . . 33

4.2 Performance measures for a SIC Receivers . . . . . . . . . . . . . . . . . 34

4.3 Results . . . . . . . . . . . . . . . . . . . . . . . 35

5 Conclusion and Future Work 42

$\begin{array}{ll}\text { References } & 44\end{array}$ 


\section{List of Figures}

2.1 A cooperative network with three transmitters (Tx) and a base-station (BS)

2.2 Medium access control characteristics of a non-cooperating CDMA system with K users . . . . . . . . . . . . . . . . . . . . . . . . . . . . . 14

2.3 Block diagram showing direct-sequence spread-spectrum system . . . . . . . 15

2.4 A DS-CDMA signal is generated by multiplication of a user data signal by a code sequence . . . . . . . . . . . . . . . . . . . 15

3.1 Medium access control for the space-time cooperative protocol . . . . . . . . 22

3.2 Medium access control for asynchronous CDMA cooperative protocol . . . . 24

3.3 Outage probability performance comparison between the decorrelator and the Laneman's STC under high-SNR approximation assumption for a 4-user system 28

3.4 Block diagram showing the implementation of the successive interference cancellation $(\mathrm{SIC})$ receiver . . . . . . . . . . . . . . . . .

4.1 Information-outage probability performance of the proposed asynchronous underloaded CDMA uplink $(K<N)$ with $N=4, K=m=3$ without using high-SNR approximations. The spectral efficiency used as the threshold is $R=1 \mathrm{bit} / \mathrm{sec} / \mathrm{Hz}$. Also shown for comparison are the Laneman's synchronous STC $(m=4)$ and decorrelator outage probabilities with a high SNR approximation.

4.2 Information-outage probability performance of the proposed asynchronous fully-loaded CDMA uplink $(K=N)$ with $N=4, K=m=4$ without using high-SNR approximations. The spectral efficiency used as the threshold is $R=1 \mathrm{bit} / \mathrm{sec} / \mathrm{Hz}$. Also shown for comparison are the Laneman's synchronous STC $(m=4)$ and decorrelator outage probabilities with a high SNR approximation.

4.3 Information-outage probability performance of the proposed asynchronous over-loaded CDMA uplink $(K>N)$ with $N=4, K=m=5$ without using high-SNR approximations. The spectral efficiency used as the threshold is $R=1 \mathrm{bit} / \mathrm{sec} / \mathrm{Hz}$. Also shown for comparison are the Laneman's synchronous STC $(m=4)$ and decorrelator outage probabilities with a high SNR approximation. 
4.4 Comparison of Information-outage probability performance of the proposed asynchronous overloaded CDMA uplink $(K>N)$ with $N=4, K=m=5$ for different users of the decorrelator with SIC without using any SNR approximations. The spectral efficiency used as the threshold is $R=1 \mathrm{bit} / \mathrm{sec} / \mathrm{Hz}$. Also shown for comparison is the Laneman's synchronous STC $(m=4)$. . .

4.5 Comparison of Information-outage probability performance of the proposed asynchronous overloaded CDMA uplink $(K>N)$ with $N=4, K=m=5$ for different users of the MMSE detector with SIC without using any SNR approximations. The spectral efficiency used as the threshold is $R=1 \mathrm{bit} / \mathrm{sec} / \mathrm{Hz}$. Also shown for comparison is the Laneman's synchronous STC $(m=4)$. . . 


\section{Notation}

We use the following notation and symbols throughout this thesis.

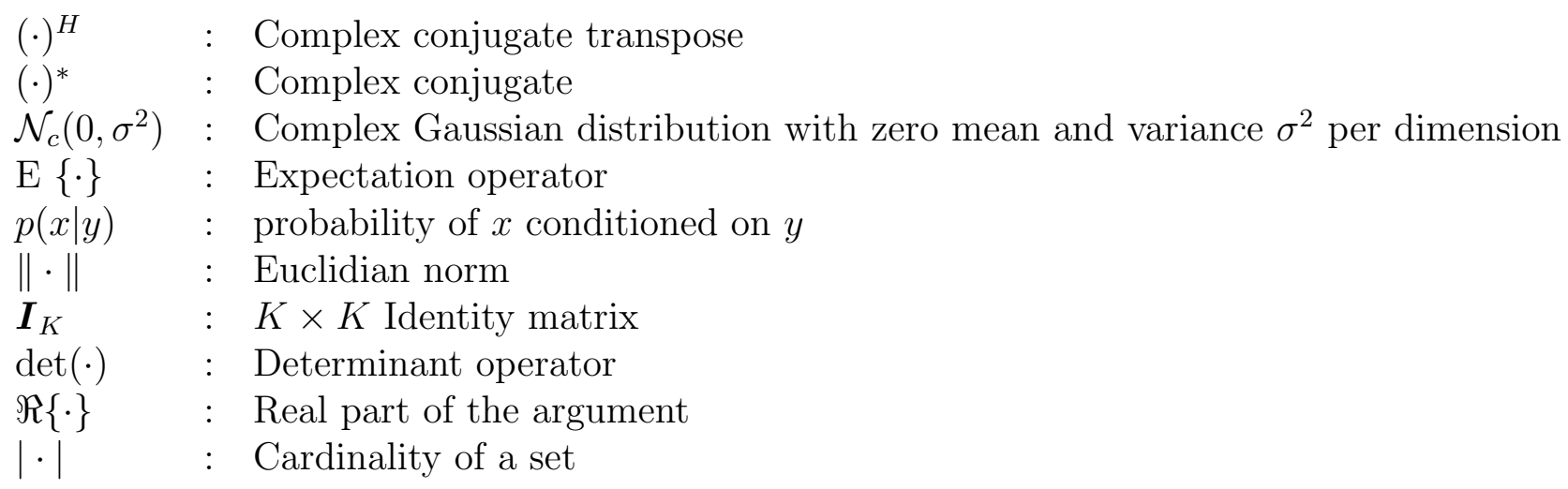

Bold uppercase letters denote matrices while bold lower case letters denote vectors. 


\section{Chapter 1}

\section{Introduction}

There has been rapid growth in wireless communications since the introduction of the cellular telephones, and this is expected to grow further through the development of new schemes and technologies. These new technologies overcome the limitations of the previous techniques used and also help meet the high data rate and the high reliability communication requirements. The use of wireless handheld devices has increased and these users demand the services like streaming high resolution videos and images wirelessly. And all these services require high data rates inorder of Gbps and low latencies. New technologies like the emerging 3G LTE [2], 4G [3], WiMax [4] and WiGig [5] help satisfy these needs of the users.

Fading is a key factor that distinguishes the wireless communications from the wireline communications. Fading is often considered as a random process and it is the channel phenomenon which may be the time variation of the channel strengths due to the smallscale effect of multipath fading or may be a larger-scale effect such as path loss via distance attenuation and shadowing by obstacles [6]. There are many ways of improving the SNR and one of them is to increase the transmit signal power during a deep fade and decrease it if the fading is not deep. This might result in large peak to average power ratios and this solution is very impractical in a mobile environment because it is difficult to develop the mobile device's battery with huge dynamic ranges of power. Another method is to transmit the signal only if the fading the not deep and if there is a fading in the channel then stop transmitting. This method requires several assumptions which are estimating the channel so that the transmitter knows the fading conditions and also knowing the delays caused at the 
mobile station. Both the above methods are not quite useful in a cellular uplink. A possible solution is to transmit signal through multiple paths each of which fades independently and there by increasing system's reliability since atleast one of those multiple signal paths might have a less fade. This is the concept of diversity and this can be obtained in one of these ways: time, frequency and space. Diversity over time is obtained by coding the information and then dispersing these coded symbols in different coherence periods so that different parts of the codewords experience independent fades. Using a frequency selective channel could help in achieving the frequency diversity. The spatial diversity can be obtained by using multiple antennas at the transmitter or receiver (also know as MIMO systems) provided the separation between the antennas is more and this helps in creating independent signal paths. The space time codes introduced in [7] are used to exploit the spatial diversity created by multiple antennas. [8] uses space time orthogonal codes provide full diversity with lower encoder/decoder complexity. Space time trellis codes used in [7], provide large coding gains but are difficult to design. Beamforming is another technique to control the SNR/fading through the use of multiple antennas.

MIMO systems are now being used in many communication standards like 802.11n, 4G and WiMAX. These MIMO systems are getting popular as they increase the spectral efficiency and also provide high data rates under the same transmit power budget and biterror-rate performance requirements [9], [10] and [11]. These performance improvements result from the diversity gain, array gain, spatial multiplexing and interference reduction capabilities of the MIMO networks [12]. Spatial Multiplexing is achieved by exploiting the structure of the channel gain matrix to obtain independent signaling paths that can be used to send independent data and there by achieving the required uncorrelated fading gains [13]. Also the spacing between multiple antennas either used at the transmitter or the receiver needs to be greater than half of a wavelength to avoid fading correlation. And for most of the mobile wireless networks the carrier frequency is not much higher than $1 \mathrm{GHz}$ or equivalently the wavelength is not smaller than $0.3 \mathrm{~m}$ [14]. Also the antennas of bigger dimensions and using a number of such antennas are not practical in the mobile nodes which are used in applications like cellular uplinks or Wireless LAN's. Moreover, using a number of base stations or using a number of repeaters in a network is costly. And a possible solution to this 
problem is to use the broadcasting property of a node in the network and using this we can allow the individual nodes of a network to cooperate on transmitting the information there by creating a virtual antenna array [15]. This antenna array would now form a network which is a 'distributed MIMO'. This idea of cooperative diversity could be applied to a cellular uplinks or any ad-hoc network.

In a wireless network with multiple nodes, the transmitted signal from a source is not just received by the destination mobile node but it is also overheard by the nodes between source and destination. So the overheard information from the other nodes can be used to achieve the diversity and this is the main principle involved in the cooperative communications. These kind of networks are based on two kinds cooperative diversity protocols: decode-and-forward and amplify-and-forward. In decode-and-forward, each relay receives the transmitted signal from source and they decode it. The decoded signal is then forwarded to the destination. Amplify-and-forward simply amplifies the received signal and then forwards it to the destination.

\subsection{Thesis Outline}

In this thesis we investigate cooperative diversity in an asynchronous code-division multipleaccess (CDMA) uplink by taking inter-user non-orthogonality (allocation of non-orthogonal channels to multiple users) into consideration. We chose the CDMA uplink because this helps to address the effects of multiuser interference in a simple way.

User cooperation is the effective way of achieving the diversity in most of the wireless networks. The concept of this cooperative communication in cellular networks was first introduced in the [15]. This work examines the outage probabilities by using an informationtheoretic model and also a two user cooperative CDMA network. The CDMA implementation had two users cooperate by transmission each bit over two successive bit intervals. In first interval, one of the users transmit a bit and in second interval, the other detects and retransmits the estimate of that bit. [16]- [17] has extended this work to a number of other cooperative diversity protocols. 
Most the previous research works on the cooperative diversity including [15] - [18] assumes the use of the inter-user orthogonality and the synchronism between the signals transmitted from different users. Both of these assumptions might bring in extra complexities in the network as they both will need the users to be accurately coordinated in a cellular network. Some of the problems regarding user orthogonality and synchronous issues has been later addressed in [19] and [20]. The system in [19] assumes very loose synchronous constraints and the key idea of this work is to have the nodes simply echo the leaders transmission operating as active scatterers.

A distributed delay diversity approach was used in [20] to achieve the diversity gains promised by distributed spacetime codes. The work in [21] addresses most the of the multiple access interference (MAI) issues and also analyses the information-outage probability performance in multi-user cooperative diversity scheme under non-orthogonal channel allocation and the asynchronous communication constraints. However the decorrelator approach used in this work is highly suboptimal in handling asynchronism and non-orthogonality. In the outage probability of analysis [21], it is seen that there is a large gap between the results shown and the interference free case [22]. The SNR loss incurred with respect to [22] in this method could be reduced by using alternative receiver structures. Here we try to close the gap between the outage probability performance of [22] and [21] by using the MMSE receivers and also a employ a successive interference cancellation techniques for both the doecorrelator detector in [21] and also the proposed MMSE detector.

In Chapter 2, we present an overview of the wireless communications addressing the issues of fading and also discussed are some ways to mitigate the affects of large scale fading and small scale fading. We also review the various diversity techniques, particularly the spatial diversity which is of our interest. We then brief the MIMO systems and discuss on how these systems achieve good diversity gains. We also talk about the the concept of cooperative diversity and its use in the cellular uplink. Also reviewed is the CDMA technique and we present a synchronous CDMA system model as well. In Chapter 3, we first review the non-cooperative asynchronous CDMA systems and then we present our system model used in this thesis. In the next section we first present the system model of the Laneman's distributed STC [22] and in the succeeding section we present our system model and then 
review the decorrelator detector used in [21]. We later propose our MMSE and SIC schemes.

In Chapter 4, we will analyze all of the outage probability performance curves. Finally, Chapter 5 provides the conclusions and some suggestions for future work. 


\section{Chapter 2}

\section{Wireless Communication Systems Overview and Background}

\subsection{Representation of Signals in a Communication Sys- tem}

The purpose of a communication system is to transfer the information in form of a signal from one point in space and time, called the source, to another, the destination by means of a physical medium called as a channel. In most of the cases the spectral characteristics of the information signal do not directly match the spectral characteristics of the communication channel and hence the signal cannot be directly transmitted over by the channel. Therefore many of the signals in the communication systems are real bandpass signals and these are a result of modulating a baseband signal by a carrier or from filtering a deterministic or random signal with a bandpass filter. A bandpass signal, $x(t)$ whose frequency contents centered around carrier frequency ' $f_{c}$ ' $\mathrm{Hz}$ can be represented as follows:

$$
x(t)=\operatorname{Re}\left[s(t) e^{j 2 \pi f_{c} t}\right]
$$

where $s(t)$ is the lowpass equivalent of the original bandpass signal $x(t)$. The $s(t)$, which is the information bearing signal is extracted at the destination by means of a demodulator which basically reverses the function of modulation. 


\subsection{The Wireless Channel}

The Additive White Gaussian Noise (AWGN) channel is considered to be the simplest mathematical model for a communication channel. In this channel model, the transmitted signal $x(t)$ is corrupted by an additive random noise process $n(t)$. The additive noise may arise from the electronic components and amplifiers at the receivers and this type of noise is characterized statistically as a Gaussian noise process. This channel is mathematically given as follows:

$$
r(t)=x(t)+n(t)
$$

where $r(t)$ is the received signal and also $n(t)$ is a zero mean gaussian noise with power spectral density of $N_{0} / 2$. The receiver observes the signal $r(t)$ and then makes an optimal decision on the $x(t)$. The optimal decision is the one which results in a minimum error probability. This AWGN channel is also considered to an ideal model for the wireless communication systems.

\subsubsection{Fading}

A practical wireless channel model considers the time and frequency selective distortions in the received signal strength called as fading. There are several mechanisms behind this fading and some of them are free space path loss, reflection, scattering and diffraction. So in a typical urban environment, a signal transmitted from the source is most likely to collide with multiple objects which produces multiple copies of the transmitted signal which are reflected, diffracted or scattered. These additional copies of the transmitted signal are called as multipath signal components and at the receiver all these components are combined with the transmitted signal which is most likely to produce distortions in the received signal. The fading is generally characterized into two types: Large scale and Small scale.

\section{Large Scale Fading}

Large scale fading results in path loss and shadowing where in there are large variations in the received signal power as the distance between the transmitter and receiver increases. 
In this thesis we assume that all of the large scale effects have been resolved through the power control techniques.

\section{Small Scale Fading}

Small scale fading refers to abrupt changes in the path-loss for small separation distances between transmitter and receiver. This type of fading takes place due to the time-spreading of underlying pulses withing the signal and also due to time variant nature of the channel. If the number of multipath components is large and there is no line-of-sight (LOS) signal component, then the envelope of the received signal follows a Rayleigh distribution. And it at all the LOS component is large, then the envelope will follow a Rician distribution.

Depending on the relation between the signal parameters and the channel parameters different transmitted signals will undergo different types of fading. In time domain, the most commonly used parameters are maximum excess delay, $T_{m}$ and the rms delay spread, $\sigma_{\tau}$. The maximum excess delay is the delay between the first and last arrivals of the diverse signal components. Based on this $T_{m}$ and the symbol time $T_{s}$, two categories of fading are derived:

\section{Flat Fading}

In this case the maximum excess delay is less than the symbol period and hence there is only one single resolvable path which itself is composed of many unresolvable multipath components. This causes a reduction in the signal to noise ratio.

\section{Frequency Selective Fading}

Here the maximum excess delay is greater than the symbol period and this results in multiple resolvable signal paths. This causes the channel induced Inter-Symbol Interference (ISI), where the received symbol experiences interference from other symbols that have been delayed by the multipath. 
Now, in the analogous frequency domain, the important parameter is coherence bandwidth, $B_{m}$. This is range of frequencies over which channel has constant gain and linear phase response. Now also according to the $B_{m}$ and the symbol rate $B=1 / T_{s}$, the channel is recognized as a flat fading $B_{m}>B$ or a frequency selective fading channel $B_{m}<B$.

The other important parameter which describe the time dispersive nature of the channel due to the relative motion between the transmitter and the receiver is the Doppler Spread, $B_{d}$. Doppler spread is the measure of the spectral broadening caused by the time rate of change of the mobile radio channel and is defined as the range of frequencies over which the received Doppler spectrum is essentially non-zero. And based on this doppler spread and the symbol rate, $B$, the fading is categorized as fast fading, $B_{d}>B$ and slow fading, $B_{d}<B$ channels respectively. In the time-domain, the Coherence time, $T_{c}$ is the dual of the doppler spread and this measures the time duration over which the channel has an essentially invariant response. And based on this $T_{c}$ and symbol period $T_{s}$, the channel is fast fading if $T c<T_{s}$ and is slow fading if $T_{c}>T_{s}$.

In order to model slow frequency non-selective fading channels, the Block fading channels were introduced. Now in this block fading, the channel fading coefficient remains constant over a block of data. And through out this thesis we will consider a flat fading block channel with the Rayleigh distribution.

\subsection{Diversity}

Diversity is one of the important techniques that mitigates the effects of fading by combining the independently faded signal paths. The main cause of the poor performance of the communication system is that if we use a single signal path, there is a relatively high probability that signal path may be in a deep fade, in the order of $1 /$ SNR [6]. So if we could send the same signal over multiple independent paths then it is possible that not all of them can be in deep fades at a single point of time. This is the key technique behind the diversity. There are many ways of achieving the diversity which include time diversity, frequency diversity and space diversity. Time diversity is achieved by averaging the fading of the channel 
over the time. Frequency diversity achieves the independent fades by sending information over multiple carrier frequencies which are separated by at least the coherence bandwidth of the channel. Analogously, the spatial diversity or else known as antenna diversity make use of multiple antennas at the transmitter and/or receiver and by using an appropriate separation between the antennas, independent signal paths are created.

\subsubsection{MIMO}

The basic idea of MIMO systems is to improve the quality (in terms of bit error rate (BER) ) and the data rate by using the multiple transmitter/receiver antennas. It was first explored in [23] that this use of multiple antennas through MIMO systems for increasing capacity. Later, the works [10] [24] [7] [25] have shown that MIMO systems have clearly an advantage of higher spectral efficiency and improved reliability over the SISO systems. Two important methodologies used in MIMO are spatial diversity and spatial multiplexing. Spatial diversity is an approach where signal copies are transmitted from multiple antennas or received at multiple antennas [8] [7] [26]. Spatial multiplexing on the other hand is an approach where the signal stream is divided into independent multiple substreams and each one of them is transmitted on a different transmit antenna [24] [9]. The spectral efficiency is quantified by the multiplexing gain and the reliability is by the diversity gain. The diversity order or diversity gain of a MIMO system is also defined as the number of independent receptions of the same signal. Consider a MIMO system with $M$ transmit antennas and $N$ receive antennas. Then that system is said to have full diversity (i.e. maximum diversity) gain equal to $M N$. Fully exploiting both the multiplexing and diversity gains together is not possible because a MIMO system cannot achieve both the diversity gain and spatial multiplexing gain simultaneously. This tradeoff between the diversity and spatial multiplexing is also known as Diversity-Multiplexing tradeoff and this was discussed in $[27]$.

The spatial multiplexing in MIMO systems is exploited by the use of Bell Laboratories Layered Space-Time (BLAST) architecture proposed in [24] and Vertical-BLAST or VBLAST in [28] which is a simplified version of BLAST scheme in [24]. This BLAST scheme 
is capable of supporting high data rates which are linearly proportional to the number of the transmitting and receiving antennas. In a typical BLAST system, a single data stream is demultiplexed into $M$ substreams and these substreams are then transmitted over $M$ transmitting antennas. The receiver antennas receive the substreams which are then separated by means of sequential interference nulling/cancellation. Although this scheme achieves a high channel capacity, it suffers from a poor diversity gain. In a given i.i.d Rayleigh fading channel with $M$ transmitting antennas and $N$ receiving antennas (provided $M \leq N$ ), the first detected substream has a diversity gain of only $M-N+1$ which will create a bottleneck on the overall performance and this effect could be mitigated by the use of optimal ordering techniques.

The diversity gains are explored by the Space-time coding techniques [8] [7] [26] and these transmit the same stream through all the transmit antennas which is different from BLAST which transmits different streams through different antennas. The Space time block codes (STBC) are the most dominant form of the space-time codes used and the Alamouti's scheme was the first STBC. [8] uses two transmit antennas and $N$ receive antennas and can achieve a maximum diversity order of $2 N$. Space time trellis codes are the other type of space-time codes used in case of multiple transmit and receive antennas.

\subsubsection{Cooperative Diversity}

The MIMO system uses multiple antennas at the transmitter and/or receiver and the physical separation between the antennas must be high enough to generate the independent fading paths. The classical rule for the antenna separation is that the distance between two antennas must be greater than half of the wavelength. The wider antenna separations are possible at the base station but it is not possible in the smaller mobile units like cell phones. So a new form of spatial diversity called as Cooperative diversity [15] [29] [18] was introduced.

Cooperative diversity takes the advantage of the basic broadcasting property of the wireless network. When we transmit a message from a wireless node to the base station, the nearby neighboring nodes to the source might also overhear the message. This overheard 
information is generally ignored in a non-cooperative network. But we can actually make the relays forward that message to the base station and then the base station will receive multiple copies of the transmitted message, thereby exploiting the diversity. We could avoid the multiple access interference problems by making the time slots of transmission from source and the relay orthogonal. We could achieve this orthogonality between the source and relay signals by either using the orthogonal spreading codes or the orthogonal space-time codes. Two of the most important relaying protocols used in the cooperative networks are Amplify and forward (AF) and Decode and forward (DF). In the AF scheme, the nodes acting as

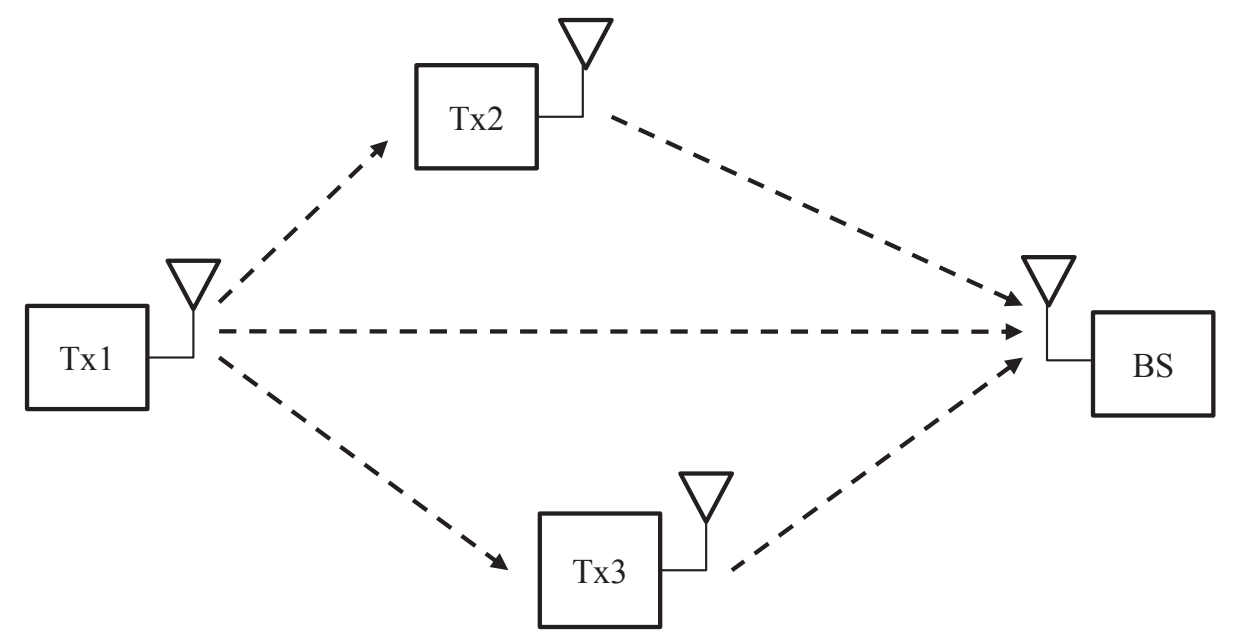

Figure 2.1: A cooperative network with three transmitters (Tx) and a base-station (BS)

relays overhear the information from the source, and this information is simply amplified by the relay. The amplified information is then sent to the destination. On the other hand, in a DF scheme, the relay tries to first decode the information it has overheard and then it forwards that decoded information to the destination. In case of the AF scheme, there is not any kind of demodulation or decoding done with the information it receives. And in most of the cases, the AF relay would be transmitting a noisy signal to the destination. This is not the case with DF scheme since it does decode the information it receives over the fading channel. But this process might involve some delays since it takes some time to decode and then retransmit the data. So each of the schemes has it own share of advantages and disadvantages. The details analysis of these protocols are given in [16]- [17]. Throughout 
this thesis we will be using a decode and forward relaying scheme.

\subsection{Multiple Access Techniques}

Given a multiuser system, there are many users trying to user the resources at the same time and hence the system resources must be shared among the multiple users. One of the several resources shared is the finite radio spectrum and this is basically done using the Multiple Access methods. Multiple access also refers to assigning the signaling dimensions to a multiuser system [13]. The multiple access methods behave differently in different multiuser channels. And a multiuser channel is generally categorized into two types: the downlink channel and the uplink channel. The downlink channel has one transmitter which basically broadcasts information to many receivers and this channel is also called as the broadcast channel or the forward channel. The uplink channel which is quite inverse of the downlink channel, has many transmitters sending the information to one receiver. And this uplink is also called as the multiple access channel or the reverse channel.

Multiple access methods work by dividing the signaling dimensions in to channels and then these channels are assigned to the users. Based on the way it divides the signal space, the multiple access methods are classified into time-division multiple access (TDMA), frequencydivision multiple access (FDMA) and code-division multiple access (CDMA). In FDMA, the signals from different users are assigned different channels/frequencies. So at a given time each channel is assigned only to one user. In a TDMA system, a same frequency spectrum is used for all of the users but the number of users it can accommodate in a same band of frequencies is high since it assigns the channel based on the time slots. In a CDMA system, each user is assigned a pseudo-random sequence by using which the users can transmit simultaneously across the entire frequency band. This pseudo-random sequence is also called as pseudorandom noise $(\mathrm{PN})$.

\subsubsection{CDMA}

The CDMA technique has been the most popular multiple access methods which is still being used. It works by using a different signature waveforms or codes for every user. Then 


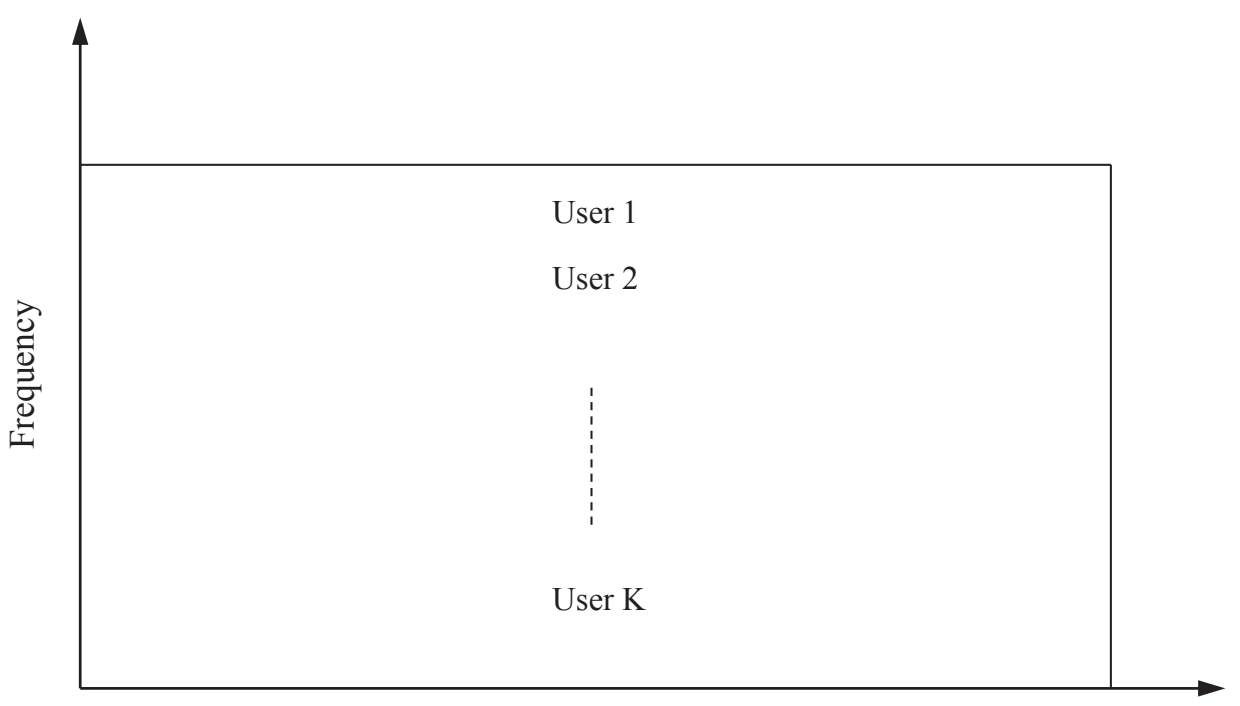

Time

Figure 2.2: Medium access control characteristics of a non-cooperating CDMA system with $\mathrm{K}$ users

each user transmits its data by modulating its own signature waveform as in a single user communication system. These signature waveforms can be either orthogonal or non-orthogonal. CDMA is an application of the spread-spectrum techniques [30]. In a spread spectrum scheme, the signal occupies a larger bandwidth than is necessary to send information and this increase or a spread in the bandwidth is achieved by a code which is pseudo-random. The spread spectrum signals are divided into two main groups - direct sequence spread spectrum (DS-SS) and the frequency hopping spread spectrum (FH-SS). The CDMA is form of direct sequence spread spectrum and hence DS-SS is of our interest. In the DS-SS, the data is multiplied by a high frequency $\mathrm{PN}$ sequence of $\pm 1^{\prime} s$ and then the resulting spreaded signal is transmitted via a channel. The receiver multiplies the synchronized replica of PN sequence to the received signal yielding a noisy version of the data. The block diagram of the DS-SS system is shown in Figure-2.1 and the generation of a DS-CDMA signal is shown in Figure-2.2. 


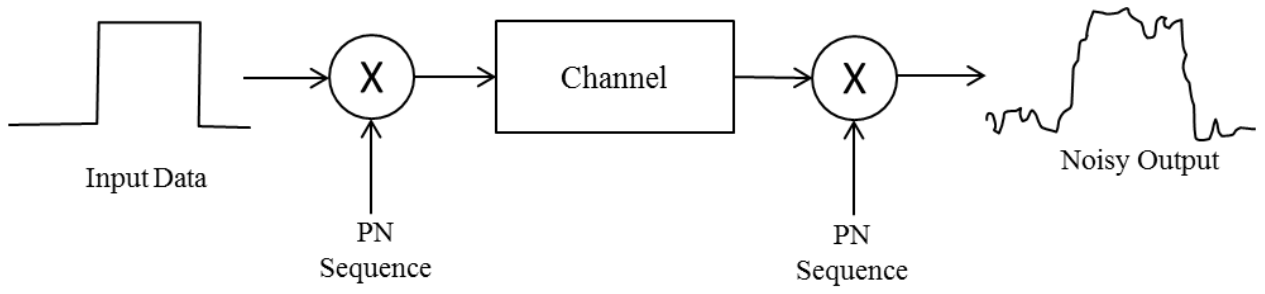

Figure 2.3: Block diagram showing direct-sequence spread-spectrum system

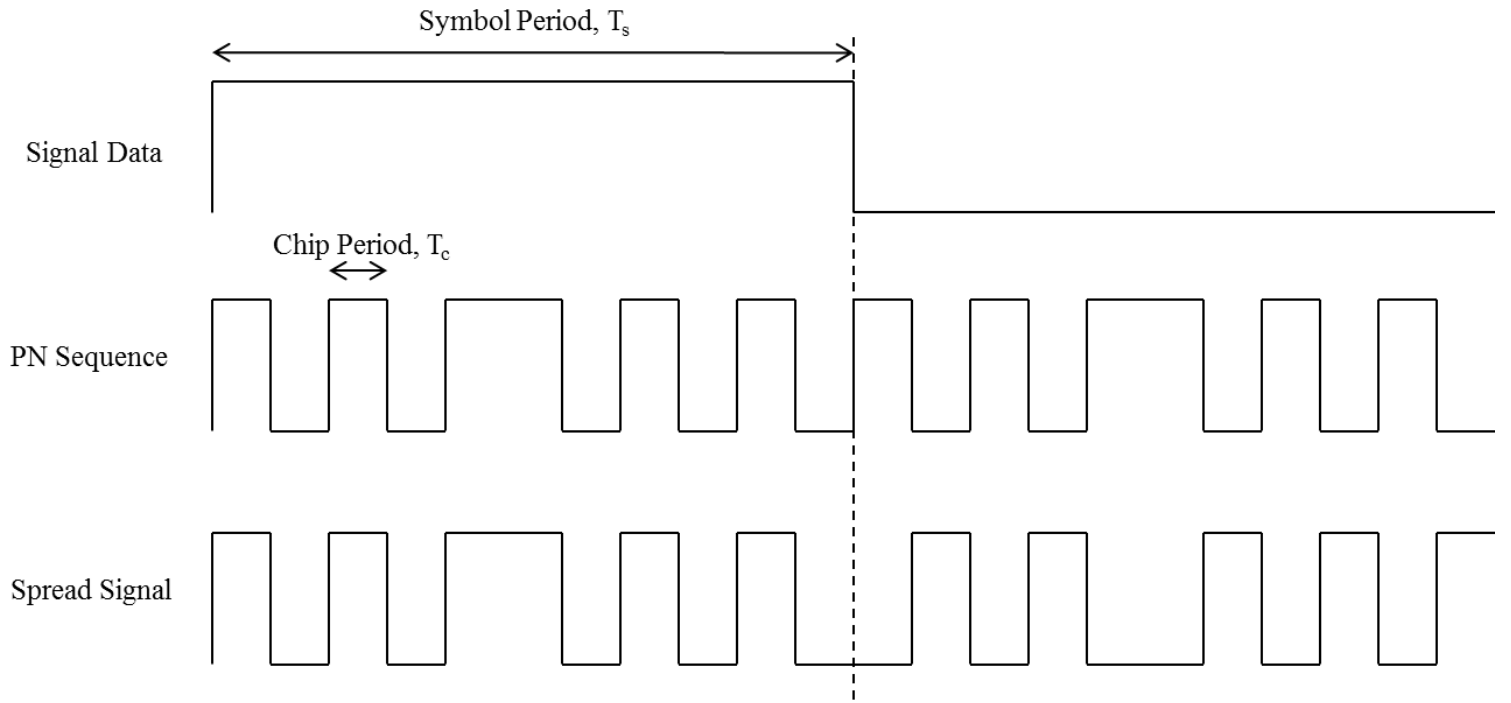

Figure 2.4: A DS-CDMA signal is generated by multiplication of a user data signal by a code sequence

\section{Discrete-Time Synchronous CDMA}

In a synchronous CDMA system the symbols from each user arrives at the receiver at same time and most often the information bit streams are aligned in time (the starting and the ending times of bits are synchronized in time). Here we will consider a basic $K$-user CDMA system with a non-fading AWGN channel. The binary information data of each user $(k)$ is BPSK symbol mapped yielding data symbols of duration $T_{s}$. Each of these data symbols $\left(b_{k}\right)$ is then modulated by the spreading sequence $s_{k}$ and the resulting signal is then transmitted. Below is the signal transmitted by the $k^{\text {th }}$ user.

$$
x_{k}(t)=A_{k} \sum_{i=0}^{M-1} b_{k}[i] s_{k}\left(t-i T_{s}\right),
$$


where $M$ is the frame size or the number of data symbols per user per frame, $T_{s}$ is the symbol interval and $A_{k}$ and $s_{k}(t)$ denote the amplitude and the spreading waveform of user $k$. The spreading waveform is given by

$$
s_{k}(t)=\sum_{n=0}^{N-1} c_{k}[n] \psi\left(t-n T_{c}\right) .
$$

In the above equation, $T_{c}$ represents the chip interval given by $T_{c}=T_{s} / N, N$ is the processing gain, $c_{k}[n] \in\{ \pm 1 / \sqrt{N}\}$ is the PN sequence and $\psi(n)$ is unit energy, baseband pulse with is supported on the interval $\left[0, T_{c}\right]$. This signal $x_{k}(t)$ propagates through a non-fading AWGN channel and the receiver gets a superposition of all the signals from $K$ users and it is given by

$$
r(t)=\sum_{i=0}^{M-1} \sum_{k=1}^{K} A_{k} b_{k}[i] s_{k}\left(t-i T_{s}\right)+n(t),
$$

where $n(t)$ is a white Gaussian noise with PSD $\frac{N_{0}}{2}$.

Now since this is synchronous case, the sufficient statistic required for the demodulation of the $i^{\text {th }}$ code bit of $k^{\text {th }}$ user is given by $y_{k}[i]$, which is a matched filter output of user $k$ in the $i^{\text {th }}$ symbol interval and is given by

$$
\begin{aligned}
y_{k}[i] & =\int_{i T_{s}}^{(i+1) T_{s}} s_{k}\left(t-i T_{s}\right) r(t) d t \\
& =\int_{i T_{s}}^{(i+1) T_{s}} s_{k}\left(t-i T_{s}\right) \times \sum_{m=0}^{M-1} \sum_{k^{\prime}=1}^{K} A_{k^{\prime}} b_{k^{\prime}}[m] s_{k^{\prime}}\left(t-m T_{s}\right)+n(t) d t \\
& =\sum_{m=0}^{M-1} \sum_{k^{\prime}=1}^{K} A_{k^{\prime}} b_{k^{\prime}}[m] \int_{i T_{s}}^{(i+1) T_{s}} s_{k}\left(t-i T_{s}\right) s_{k^{\prime}}\left(t-m T_{s}\right) d t+n_{k}(i),
\end{aligned}
$$

where

$$
n_{k}[i]=\int_{i T_{s}}^{(i+1) T_{s}} n(t) s_{k}\left(t-i T_{s}\right) .
$$

Note that $s_{k}(t)$ is a deterministic spreading waveform normalized to have unit energy and hence

$$
\left\|s_{k}(t)\right\|^{2}=\int_{-\infty}^{\infty} s_{k}^{2}(t) d t=1
$$


The spreading waveforms are assumed to be zero outside the interval $\left[0, T_{s}\right]$ and also $\psi(t)$ is supported only in $\left[0, T_{c}\right]$ and hence,

$$
\int_{i T_{s}}^{(i+1) T_{s}} s_{k}\left(t-i T_{s}\right) s_{k^{\prime}}\left(t-m T_{s}\right) d t \propto \delta_{i, m} .
$$

Now,

$$
\begin{aligned}
y_{k}[i] & =\sum_{k^{\prime}=1}^{K} A_{k^{\prime}} b_{k^{\prime}}[i] \int_{0}^{T_{s}} s_{k}(t) s_{k}^{\prime}(t) d t+n_{k}[i] \\
& =\sum_{k^{\prime}=1}^{K} A_{k^{\prime}} b_{k^{\prime}}[i] \rho_{k, k^{\prime}}+n_{k}[i],
\end{aligned}
$$

where $\rho_{k, k^{\prime}}$ is the crosscorrelation coefficient between user $k$ and $k^{\prime}$ and is given by

$$
\rho_{k, k^{\prime}}=\left\langle s_{k}(t), s_{k^{\prime}}(t)\right\rangle \triangleq \int_{0}^{T_{s}} s_{k}(t) s_{k}^{\prime}(t) d t
$$

Now if we consider user 1 as our interest, then the corresponding matched filter output of user 1 is given by

$$
y_{1}[i]=A_{1} b_{1}[i]+\sum_{k=2}^{K} A_{k} b_{k}[i] \rho_{1, k}+n_{k}[i] \quad i=0,1, \ldots . ., M-1
$$

In the above expression the first term is the signal of our interest and the second term represents the multiple access interference caused by other user's signals. Note that the inter-symbol interference is not present since the signature waveforms are assumed to be zero outside the interval $\left[0, T_{s}\right]$.

Stacking up the matched filter outputs from all $K$ users yields the following vector form:

$$
\boldsymbol{y}[i]=\boldsymbol{R} \boldsymbol{A} \boldsymbol{b}[i]+\boldsymbol{n}[i],
$$

where $\boldsymbol{y}[i]=\left[y_{1}[i] y_{2}[i] \ldots y_{K}[i]\right]^{T}, \boldsymbol{b}=\left[b_{1} b_{2} \ldots b_{K}\right]^{T}, \boldsymbol{A}=\operatorname{diag}\left\{A_{1}, A_{2}, \ldots, A_{K}\right\},[\boldsymbol{R}]_{i j}=\rho_{i, j}$ and $\boldsymbol{n} \sim \mathcal{N}_{c}\left(\mathbf{0}, N_{0} \mathbf{I}\right)$. So now it is the job of detector to use the above sufficient statistic to estimate $b_{k}[i]$ for $k^{\text {th }}$ user using $\boldsymbol{y}[i]$ and the spreading codes which are know to the receiver.

\section{Discrete-Time Asynchronous CDMA}

An asynchronous CDMA system consists of a number of users received at the receiver with different symbol intervals. Therefore in an asynchronous channel, the users are labeled 
by their time of arrival [31] and hence $0 \leq \tau_{1} \leq \tau_{2} \leq \ldots \ldots \tau_{\ell} \leq T_{s}$. So now the baseband received signal $r(t)$ can be written as

$$
r(t)=\sum_{i=0}^{M-1} \sum_{k=1}^{K} A_{k} b_{k}[i] s_{k}\left(t-i T_{s}-\tau_{\ell}\right)+n(t) .
$$

And similar to as we have shown for the synchronous case, the sufficient statistic is obtained and is given by the following equation:

$$
y_{k}[i]=\int_{-\infty}^{\infty} s_{k}\left(t-i T_{s}-\tau_{\ell}\right) r(t) d t
$$

Using the vector notation, the matched filter can be written as follows

$$
\boldsymbol{y}[i]=\boldsymbol{R} \boldsymbol{A} \boldsymbol{b}[i]+\boldsymbol{n}[i]
$$

All the parameters in the above equation are similar to equation (2.16) except for the crosscorrelation matrix which is made up of following terms

$$
\rho_{l, k}^{l^{\prime}, k^{\prime}}=\int_{-\infty}^{\infty} s_{k}\left(t-i T_{s}-\tau_{\ell}\right) s_{k^{\prime}}\left(t-i T_{s}-\tau_{\ell^{\prime}}\right) d t
$$

In [32], the first optimum multiuser detector was developed for a asynchronous Gaussian multiple access channel which is a maximum likelihood sequence detector. It was seen that for this method in [32], the complexity of the system is increased exponentially with the increase in number of users. And a system of linear suboptimal detectors like decorrelator and MMSE detector were introduced in [33]-[34] which are less complex. The decorrelator receiver is analogous to a zero forcing equalizer which removes the MAI but at an expense of enhancing noise. MMSE detector performs slightly better than decorrelator at low SNR and is identical to decorrelator at high SNR. Another approach was introduced to remove the multiuser interference by employing a interference cancellation. Successive interference cancellation (SIC) was first addressed in [35] and later in [36]. The method in [36] subtracts the previously decoded users interference from the matched filter output for the current user and it uses this decision statistic to decodes the bit of the current user.The parallel multistage iterative approach was given in [37] and this method uses the previous stage bit estimates to cancel the interference in the current user in the current stage. [38] describes a blind adaptive scheme for multiuser detection. In this paper both the decorrelator and 
MMSE detector were realized only with prior knowledge of the signature waveform and the timing of the user of interest. And in this thesis we will use and compare the decorrelator, MMSE and SIC detectors. 


\section{Chapter 3}

\section{Cooperative Asynchronous CDMA Uplink}

In a synchronous CDMA system, all the mobile units transmit signals to the base station which are synchronized to a fraction of chip-time interval. These systems make use of the orthogonal codewords to achieve higher spectral efficiency. This synchronous transmission might be useful in some case but in a cellular uplink where mobile units transmit information to base station randomly, this technique might not work which is where the asynchronous systems are used. In an asynchronous CDMA system, a number of transmitters send the information to base station in irregular time intervals and this property makes the system more practical and flexible to use. This property also makes asynchronous CDMA's more advantageous since it helps to effectively use the available frequency spectrum and also increases the flexibility in sharing the resources in mobile applications. In this chapter, we first discuss the Laneman system model [22] and the space time coded protocol used in that model. The authors in [22] use a orthogonal FDMA system. The perfectly orthogonal systems are difficult to implement without sacrificing the degrees of freedom. [21] makes use of a asynchronous CDMA and it also considers the non-orthogonality between the users. The use of inter-user nonorthogonality and the asynchronism bring in the extra complexities which include multiple access interference and inter-symbol interferences and all these effects have to be taken care of when we design our system. Later in the next section we present our system model which is similar to that used in [21]. We first consider the decorrelator receiver 
that was used in [21] and obtain its results so that we can compare them with Laneman's. We then change the receiver to our proposed MMSE detector and later extend that design by using a successive interference cancellation.

\subsection{Laneman's Distributed Space-Time Coded Coop- erative Diversity Protocol}

\subsubsection{System Model}

Laneman's protocol in [22] considers a wireless network with a total of $m$ users and each of these users have their own information to be transmitted to the base station denoted by $d$. Each source terminal $s$ transmits information via $m-1$ terminals which are relays. The relays considered are of type decode and forward and hence these are required to fully decode the source message first and then transmit a re-encoded message to the destination. All the relays which are able to decode the message successfully are together called as a decoding set $\mathcal{D}(s)$. Now we consider the medium access control (MAC) requirements for this protocol. This work considers two transmission phases. In the first phase, a source terminal $s$ transmits the information, the other users neighboring $s$ will overhear the information. During the second phase, all the relays which have overheard the source information will simultaneously transmit the information on the same sub-channel using an appropriate distributed spacetime code (STC). Hence now each terminal transmits in $1 / 2$ the degrees of freedom ${ }^{1}$ In the first phase, the signal received at each relay $r$ is given by

$$
y_{r}[n]=\alpha_{s, r} x_{s}[n]+z_{r}[n],
$$

where $x_{s}[n]$ is the signal transmitted by source $s, \alpha_{s, r}$ is the channel fading coefficient between the source and the relay with a variance of $1 / \lambda_{s, r}$ and $z_{r}[n]$ denotes the receiver noise and other forms of interference in the system. Note that the above information is same information which is also received at the base station $d$.

\footnotetext{
${ }^{1}$ Degrees of freedom in a communication channel gives information about the amount of resources available for transmission.
} 


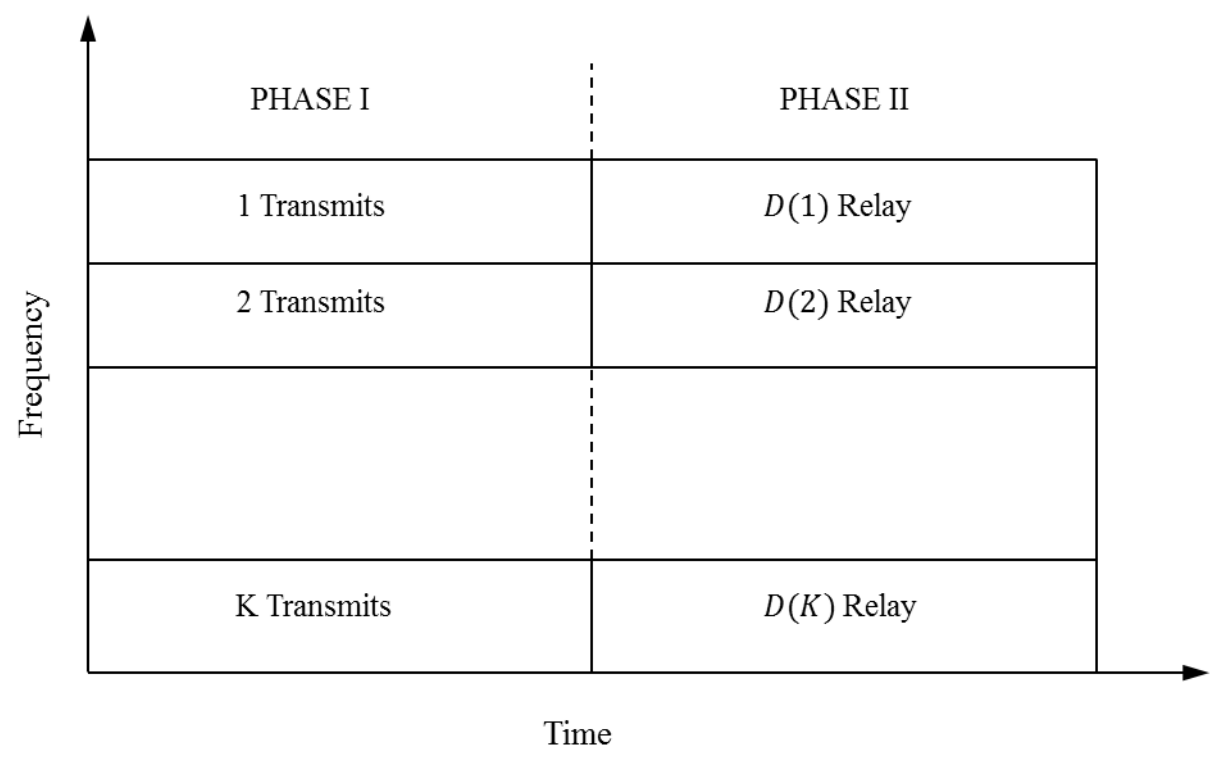

Figure 3.1: Medium access control for the space-time cooperative protocol

Now during the second phase, all of the relays transmit on a same subchannel(frequency band) and hence the destination receives the following signal:

$$
y_{d}[n]=\sum_{r \in \mathcal{D}_{(s)}} \alpha_{r, d} x_{s}[n]+z_{d}[n]
$$

And now we define the transmit SNR and the spectral efficiency $R$ for this protocol. For a continuous-time channel we consider that the total bandwidth available is $W$ hertz. For an equivalent discrete-time model, it is $W$ two-dimensional symbols per second. Similarly, in a continuous time-model, if we have an average power constraint of $P_{c}$ joules per second, a discrete time-model equivalently has a power constraint of $m P_{c} / W$ joules per two-dimensional symbol since each terminal will transmit with a $1 / m$ degrees of freedom in a non-cooperative system. Also for a cooperative transmission, it is known that each terminal transmits with $1 / 2$ of the degrees of the freedom and the normalized transmit SNR as seen in [22] is given by

$$
\mathrm{SNR}=\frac{P}{N_{0} W}=\frac{m P_{c}}{N_{0} W}
$$

The above SNR is based only on the transmit power constraint whereas the receiver SNR is a function of the channel fading coefficient and the transmit SNR, SNR. 


\subsubsection{Outage Probability for Distributed STC}

The outage probability is defined as the probability that the instantaneous mutual information $I$ falls below a fixed spectral efficiency $R$ [39]. This mutual information between the source $s$ and the base station $d$ is a function of decoding set $\mathcal{D}(s)$.

A random codebook which is independent and identically distributed (i.i.d) circularly symmetric and complex Gaussian is generated at the source terminal and the same codebook is used by all of the potential relays. Now the mutual information between source terminal and the base station conditioned on decoding set $\mathcal{D}(s)$ is given by

$$
I=\frac{1}{2} \log \left(1+\frac{2}{m} \operatorname{SNR}\left|\alpha_{s, d}\right|^{2}\right)+\frac{1}{2} \log \left(1+\frac{2}{m} \operatorname{SNR} \sum_{r \in \mathcal{D}_{(s)}}\left|\alpha_{r, d}\right|^{2}\right) .
$$

Since $\mathcal{D}(s)$ is a random entity, the outage probability for the channel between the source terminal and the base station is calculated by utilizing the total probability law and is given by

$$
\operatorname{Pr}[I<R]=\sum_{\mathcal{D}(s)} \operatorname{Pr}[\mathcal{D}(s)] \operatorname{Pr}[I<R \mid \mathcal{D}(s)]
$$

We know that for a relay to be included in the decoding set $\mathcal{D}(s)$, it must decode the source message fully and also it must also experience an SNR which is larger than its average to either of the source or destination. And the mutual information between source and the the relay for the i.i.d complex Gaussian codebooks is given by

$$
\frac{1}{2} \log \left(1+\frac{2}{m} \mathrm{SNR}\left|\alpha_{s, r}\right|^{2}\right) \text {. }
$$

And using the above, we have

$$
\begin{aligned}
\operatorname{Pr}[r \in \mathcal{D}(s)] & =\operatorname{Pr}\left[\left|\alpha_{s, r}\right|^{2}>\frac{2^{2 R}-1}{2 \mathrm{SNR} / m}\right] \\
& =\exp \left[-\lambda_{s, r} \frac{2^{2 R}-1}{2 \mathrm{SNR} / m}\right] .
\end{aligned}
$$

Finally it was shown in [22] that the outage probability for the system with the high-SNR approximation is given by

$$
\operatorname{Pr}\left[I_{s t c}<R\right] \sim\left[\frac{2^{2 R}-1}{2 \mathrm{SNR} / m}\right]^{m} \times \sum_{D(s)} \lambda_{s, d} \times \prod_{r \in D(s)} \lambda_{r, d} \prod_{r \notin D(s)} \lambda_{s, r} \times A_{|D(s)|}\left(2^{2 R}-1\right)
$$


where

$$
A_{n}(t)=\frac{1}{(n-1) !} \int_{0}^{1} \frac{w^{n-1}(1-w)}{(1+t w)} d w .
$$

\subsection{Proposed Asynchronous CDMA uplink}

\subsubsection{System Model}

In this thesis, we assume a cooperative system model which is similar to that which is used in [21]. So we would consider a CDMA cellular uplink with a set of users denoted by $\mathcal{S}$ and there are a total of $K$ user terminals and $|\mathcal{S}|=K$. Each of the above users transmit their own messages to the base station and also act as the relays to transmit the messages of others. $m$ denotes the number of the users which are cooperative and let the set of such cooperating users is denoted by $\mathcal{C} \subset \mathcal{S}$ and $|\mathcal{C}|=m$. A user is said to be cooperating if it tries to decode the other users transmissions and later can forward that transmission. Each of the users is assigned a spreading code with a processing gain $N$ and these are assumed to be non-orthogonal. A cooperative terminal relays the message by first decoding it. Then it re-encodes and re-spreads the message and it is then forwarded to the base station. The

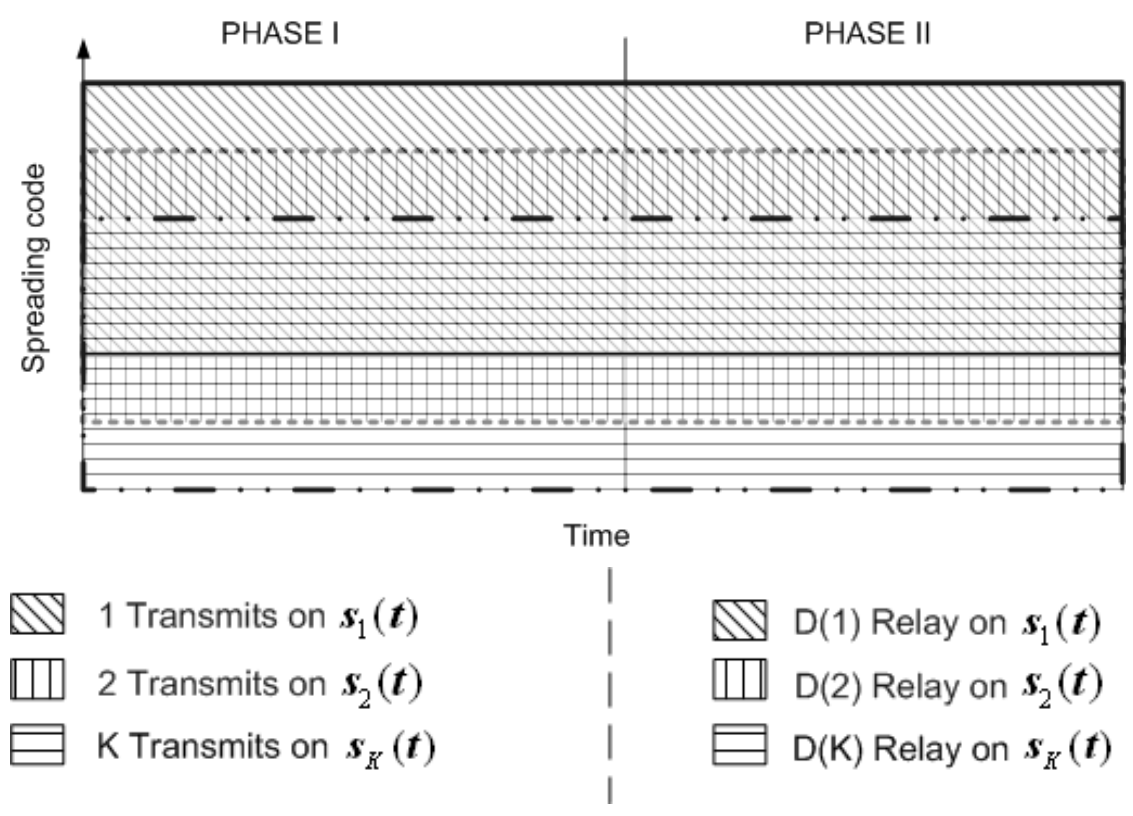

Figure 3.2: Medium access control for asynchronous CDMA cooperative protocol 
system here has full duplex relays and hence each user can transmit and receive at the same frequency. Figure 3.2 shown the channel allotments for the scheme being used in this paper. The system is basically divided into two orthogonal time phases. During the first phase every user transmits the information to the base station using a different spreading code. In the second phase users belonging to $\mathcal{C}$ decode the information and all the users that can decode the information belonging to $k$-th user. All such users belong to a set called as decoding set $\mathcal{D}(k)$ and only the users belonging to this set can be relays. The relays can now re-encode and re-spread the message and then re-transmit it to the base station and all the received message from the relays are then diversity combined at the receiver. If at all a user is not a relay, then it will continue to transmit its message in the second phase as well. Hence we achieve non-orthogonality across the channels by the use of the non-orthogonal spreading codes among the users. And since the users are allowed to transmit the messages at any time randomly, we achieve the asynchronism. This makes the system entirely practical and flexible.

The channel considered here in the proposed scheme has a flat fading Rayleigh characteristic. The use of the non-orthogonality in the spreading codes, asynchronism and also due to the fading characteristics of the channel, there is the presence of multiple access interference (MAI) and the inter-symbol interference (ISI). We will now consider the system model for the second phase since in the first phase all of the user just transmit information to base station and it is similar to a non-cooperative CDMA model. The received signal at the base station with a total of $K$ users out of which $m$ are cooperating users is given by [21]

$$
\begin{aligned}
r(t) & =\sum_{k=1}^{m} \sum_{\substack{\ell=1 \\
\ell \neq k}}^{K} \sum_{i=0}^{M-1} \alpha_{\ell} b_{\ell, k}[i] s_{k}\left(t-i T_{s}-\tau_{\ell}\right) \\
& +\sum_{k=m+1}^{K} \sum_{i=0}^{M-1} \alpha_{k} b_{k, k}[i] s_{k}\left(t-i T_{s}-\tau_{k}\right)+n(t),
\end{aligned}
$$

where $b_{l, k}[i]$ is the $k^{t h}$ user symbol which is transmitted by the $\ell^{t h}$ cooperating user and $b_{k, k}[i]$ is the $k^{\text {th }}$ user's own transmission. $\alpha_{l}$ is the flat fading channel coefficient between the user $l$ and the base station. We see that in the above expression, the first term is the result of a cooperation between the users, the second term is due to the non-cooperating users and 
the third term is the white Gaussian noise. We obtain the sufficient statistic at the receiver by match filtering the above received signal with respect to the delayed spreading waveform and it is given by

$$
\begin{aligned}
r_{k}[i] & =\int_{-\infty}^{\infty} r(t) s_{k}\left(t-i T_{s}-\tau_{l}\right) d t \\
& =\sum_{k^{\prime}=1}^{m} \sum_{\substack{l^{\prime}=1 \\
l^{\prime} \neq k^{\prime}}}^{K} \sum_{i=0}^{M-1} \alpha_{l^{\prime}} b_{l^{\prime}, k^{\prime}}[i] \rho_{l, k}^{l^{\prime}, k^{\prime}} \\
& +\sum_{k^{\prime}=m+1}^{K} \sum_{i=0}^{M-1} \alpha_{k} b_{k^{\prime}, k^{\prime}}[i] \rho_{l, k}^{k^{\prime}, k^{\prime}}+n(t),
\end{aligned}
$$

where $\rho_{l, k}^{l^{\prime}, k^{\prime}}=\int_{-\infty}^{\infty} s_{k}\left(t-i T_{s}-\tau_{l}\right) s_{k^{\prime}}\left(t-i T_{s}-\tau_{l^{\prime}}\right) d t$ is the cross-correlation between the delayed spreading waveforms. Stacking all the above matched filter responses we can write

$$
r=R A b+n
$$

where $\boldsymbol{R}$ is a function of the cross-correlations between the delayed spreading waveforms, $\mathbf{A}$ is a quasi-block diagonal matrix and is a function of only the channel gains. The elaborative definition for the cross-correlation matrix $\boldsymbol{R}$ and $\mathbf{A}$ are given clearly in the [21] and

$$
\boldsymbol{n} \sim \mathcal{N}_{c}(0, \sigma \boldsymbol{R})
$$

\subsubsection{Decorrelator Detector}

In this section we briefly explain the decorrelator detector used in the [21] for an asynchronous CDMA uplink. The channel model considered used in this thesis is identical to the one employed in the [21]. The received signal at the base station in this paper is the same as seen in equation (3.11), but the sufficient statistic at the receiver is obtained with a different matched filter than used in our proposed scheme and this doesn't hurt the performance of 
the system than that of used in (3.12). The statistic is given by

$$
\begin{aligned}
r_{l, k}[i] & =\alpha_{l}^{*} \int_{-\infty}^{\infty} r(t) s_{k}\left(t-i T_{s}-\tau_{l}\right) d t \\
& =\sum_{k^{\prime}=1}^{m} \sum_{\substack{l^{\prime}=1 \\
\ell^{\prime} \neq k^{\prime}}}^{K} \sum_{i=0}^{M-1} \alpha_{l}^{*} \alpha_{l^{\prime}} b_{l^{\prime}, k^{\prime}}[i] \rho_{l, k}^{l^{\prime}, k^{\prime}} \\
& +\sum_{k^{\prime}=m+1}^{K} \sum_{i=0}^{M-1} \alpha_{l}^{*} \alpha_{k} b_{k^{\prime}, k^{\prime}}[i] \rho_{l, k}^{k^{\prime}, k^{\prime}}+n(t),
\end{aligned}
$$

where $\rho_{l, k}^{l^{\prime}, k^{\prime}}$ is given cross-correlation between the delayed spreading waveforms which is same as seen in our proposed scheme. By stacking all of the above matched filter responses and removing the time-index the following equation is obtained:

$$
\begin{aligned}
\boldsymbol{r} & =\boldsymbol{A} \boldsymbol{R} \boldsymbol{A}^{\mathrm{H}} \boldsymbol{b}+\boldsymbol{n} \\
& =\tilde{\boldsymbol{H}}^{\mathrm{H}} \boldsymbol{b}+\boldsymbol{n}
\end{aligned}
$$

where $\boldsymbol{A}$ is the function of the channel gains $\alpha_{i}$ 's and $\boldsymbol{R}$ is the function of the crosscorrelations between the delayed spreading waveforms. Now, to the matched filter outputs shown in (3.18) a decorrelator detector is applied which yields the following:

$$
\boldsymbol{y}=(\boldsymbol{A R})^{-1} \boldsymbol{r}+\boldsymbol{v}
$$

Similar to the Laneman's model [22], the scalar channel model is obatained and is given by

$$
y_{i}=\alpha_{i}^{*} b_{i}+v_{i}
$$

\subsubsection{MMSE Detector}

In this section we obtain the similar scalar model but using a linear MMSE filter. In this case, in order to suppress the interference in the $\boldsymbol{r}_{k}[i]$ shown in equation (3.12), an instantaneous linear MMSE filter $\boldsymbol{w}_{k}(i)$ is used. And the output of the MMSE filter is given by the following equation:

$$
y_{k}[i]=\boldsymbol{w}_{k}[i]^{\mathrm{H}} \boldsymbol{r}_{k}[i]
$$




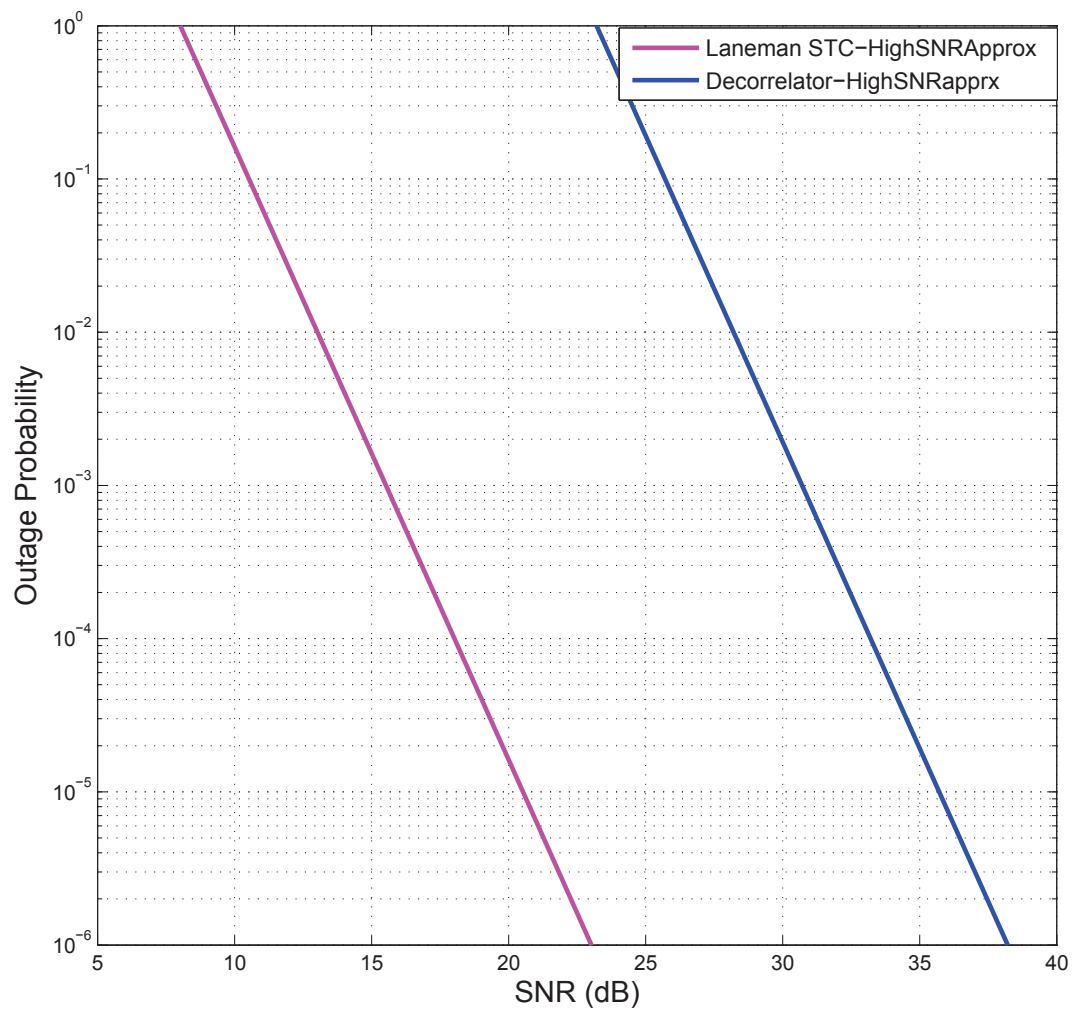

Figure 3.3: Outage probability performance comparison between the decorrelator and the Laneman's STC under high-SNR approximation assumption for a 4-user system

where $\boldsymbol{w}_{k}[i]$ is chosen to minimize the mean-square error between the $b_{k}(i)$ symbol and the filter output $y_{k}[i]$, i.e.,

$$
\begin{aligned}
\boldsymbol{w}_{k}[i]= & \arg \min _{\boldsymbol{w}} E\left\{\left\|b_{k}[i]-\boldsymbol{w}^{\mathrm{H}} \boldsymbol{r}_{k}[i]\right\|^{2}\right\} \\
= & \arg \min _{\boldsymbol{w}} \boldsymbol{w}^{\mathrm{H}} E\left\{\left\|\boldsymbol{r}_{k}[i] \boldsymbol{r}_{k}[i]^{\mathrm{H}}\right\|\right\} \boldsymbol{w} \\
& -\boldsymbol{w}^{\mathrm{H}} E\left\{\left\|b_{k}[i] \boldsymbol{r}_{k}[i]\right\|\right\}-E\left\{\left\|b_{k}[i] \boldsymbol{r}_{k}[i]\right\|\right\}^{\mathrm{H}} \boldsymbol{w},
\end{aligned}
$$

where

$$
\begin{aligned}
E\left\{\left\|\boldsymbol{r}_{k} \boldsymbol{r}_{k}^{\mathrm{H}}\right\|\right\} & =\boldsymbol{R} \boldsymbol{R}^{H}+\sigma^{2} \boldsymbol{R} . \\
E\left\{\left\|b_{k} \boldsymbol{r}_{k}\right\|\right\} & =\boldsymbol{R e}_{\mathbf{k}}
\end{aligned}
$$

where $\mathbf{e}_{\mathbf{k}}$ denotes a $K$-dimensional vector of zeros except for the $k-t h$ term. And now

$$
\boldsymbol{w}_{k}=\left[\boldsymbol{R}+\sigma^{2}\right]^{-1} \mathbf{e}_{\mathbf{k}} .
$$


It was seen in [40] that the output of a linear MMSE multi-user detector which has a residual interference plus noise can be approximated by a Gaussian distribution. Hence we have $y_{k}(i) \sim \mathcal{N}_{c}\left(\mu_{k}(i) \alpha_{k} b_{k}(i), \nu_{k}^{2}(i)\right)$. The mean and variance of $y_{k}(i)$ are given by

$$
\begin{aligned}
& \mu_{k}(i)=\left[\left[\boldsymbol{R}+\sigma^{2}\right]^{-1} \boldsymbol{R}\right]_{k, k} \\
& \nu_{k}^{2}(i)=\mu_{k}(i)-\mu_{k}(i)^{2}
\end{aligned}
$$

The above expressions are derived in a similar way as seen in [41]. And we can now obtain a scalar flat fading model similar to [22] and [21] which is given by

$$
y_{i}=\mu_{i} \alpha_{i}^{*} b_{i}+v_{i}
$$

where $v_{i}$ is enhanced zero mean Gaussian noise with variance $\nu^{2}$. Note that a similar received scalar model can be obtained in the first phase which is quite similar to one in equation (5). But the structure of $R$ might change since we do not have any users cooperating and hence $\boldsymbol{R}$ is a $K \times K$ matrix. Also in the second phase, through out this paper we consider that all the user are cooperating and hence we have $m=K$ and we get a $\boldsymbol{R}$ matrix whose size is $m^{2} \times m^{2}$. Now using the equation (14), we should be able to use the outage probability to compare this MMSE case to that of the decorrelator in [21] and [22]. The outage probability is calculated by using the average mutual information and checking whether it falls below a threshold value.

\subsubsection{SIC Receiver}

We take the system model obtained in the MMSE filter and we further try to reduce the interference by using a post-interference cancellation here. The interference cancellation involves multiple stages of canceling the noise and also the MAI from the estimated symbols. The first stage would be just obtaining the scalar model as shown in the equation (3.29). Using this model the signal-to-interference-noise ratios (SINR) are calculated and it is given by the following equation:

$$
\begin{aligned}
\operatorname{SINR} & =\frac{E^{2}\left[y_{k}(i)\right]}{\operatorname{var}\left[y_{k}(i)\right]}=\frac{\left|\alpha_{r}\right|^{2} \mu_{k}^{2}}{\mu_{k}-\mu_{k}^{2}} \\
& =\frac{\left|\alpha_{k}\right|^{2}}{1 / \mu_{k}-1} .
\end{aligned}
$$




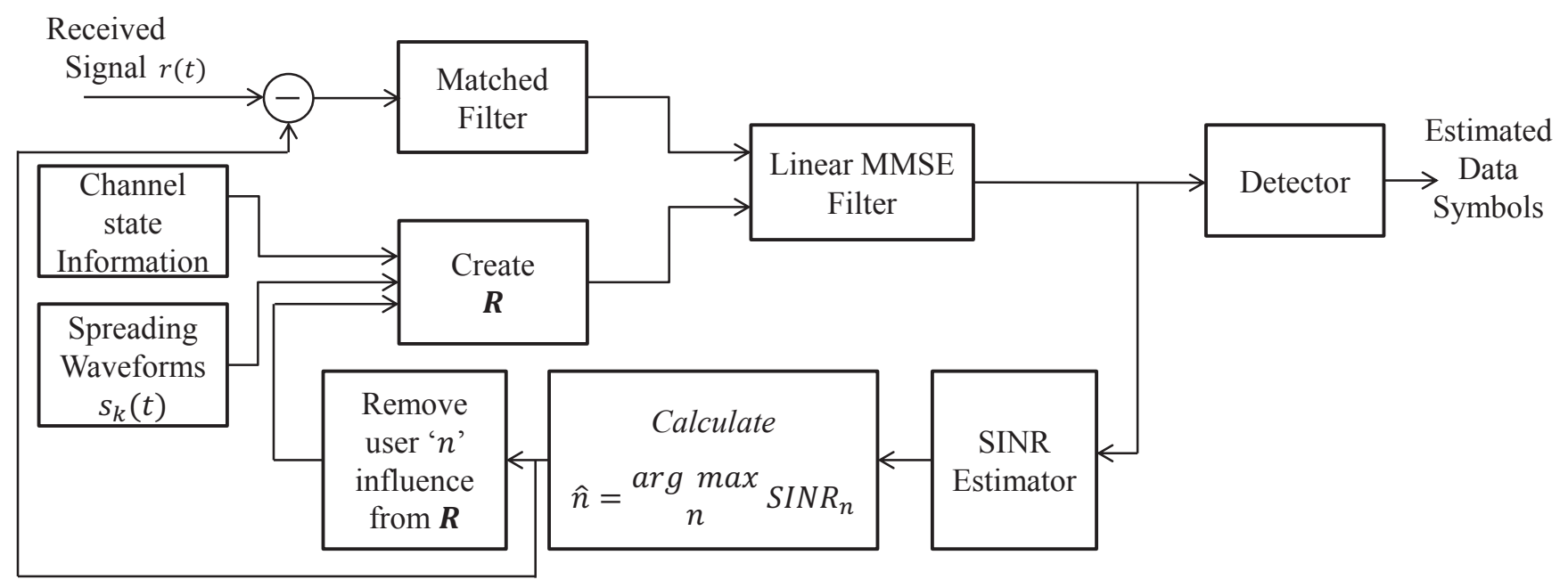

Figure 3.4: Block diagram showing the implementation of the successive interference cancellation (SIC) receiver

The user $n$ with the maximum value of the SINR is then detected and is given by

$$
\hat{n}=\arg \max _{n} \operatorname{SINR}_{n}
$$

In the second stage of the interference cancellation, the user ' $n$ ' is removed from the received signal model and the corresponding new $\boldsymbol{R}$ is then obtained. Now using this $\boldsymbol{R}$ is again passed into the instantaneous linear MMSE filter and again the SINR as calculated using the equation (3.30). Then again using these values now among the rest of the users, the user index which has the highest values of SINR is found and that user's relayed information is taken out and a new $\boldsymbol{R}$ is obtained. These stages of estimating SINR and then canceling the highest interfering user is carried out until all of the user's have been canceled. In the above figure 3.4, we see the how the interference cancellation is performed. Initially the continuous received signal at the base station is passed through matched filters and a discrete time statistic is obtained which is then passed through linear MMSE filter in the first stage. Starting from the second stage, the interference cancellation is applied and the input to the linear MMSE filter is the new discrete model obtained at every end after canceling the highest interfering user from the received model. 


\section{Chapter 4}

\section{Information-Outage Probability Analysis for MMSE and SIC Receivers ${ }^{1}$}

\subsection{Performance measures using MMSE receiver}

In this section, we will obtain the performance measure which is the mutual information for the proposed scheme. And using this mutual information we find the information-outage probability which is the probability that the average mutual information $(I)$ between the user $k$ and the base station falls below a threshold which is a fixed spectral efficiency $R$. In our proposed scheme, the relaying users belonging to the decoding set for a user transmit using the same spreading code and this information-outage probability would serve as a lower bound on the error rate of the codeword at the given same spectral efficiency $R$. So now we will obtain the mutual information expressions and there by calculate the information-outage probabilities for the underloaded CDMA, fully-loaded CDMA and the over-loaded CDMA .

\footnotetext{
${ }^{1}$ The results shown in this chapter has been published in the proceedings of the 2011 Virginia Tech wireless symposium [1]
} 


\subsubsection{Underloaded CDMA Uplink}

In an underloaded CDMA system, the number of users, $K$ is less than the processing gain, $N$ i.e., $K<N$. As mentioned earlier we assume that all the users in the system are cooperating user. We know that each user is assigned a different spreading code and there are $N$ linearly independent spreading codes available in the system. We know that channel has been divided into two orthogonal time phases and in the first phase the users transmit their own data using their spreading codes. In the second phase they send other users data using the other user's spreading codes. Hence each user effectively uses K spreading codes and hence we have $K / 2 N$ degrees of freedom in these two equally divided orthogonal phases. The mutual information between the user $k$ and the base station is given by following equation:

$$
\begin{aligned}
I_{\mathrm{u}-\mathrm{CDMA}}= & \frac{K}{2 N} \log \left(1+\frac{2 N \operatorname{SNR}}{K^{2}} \frac{\left|\alpha_{k}\right|^{2} \mu_{k}^{2}}{\mu_{k}-\mu_{k}^{2}}\right) \\
& +\frac{K}{2 N} \log \left(1+\frac{2 N \operatorname{SNR}}{K^{2}} \sum_{r \in \mathcal{D}(k)} \frac{\left|\alpha_{r}\right|^{2} \mu_{k}^{2}}{\mu_{k}-\mu_{k}^{2}}\right) \\
= & \frac{K}{2 N} \log \left(1+\frac{2 N \operatorname{SNR}}{K^{2}} \frac{\left|\alpha_{k}\right|^{2}}{1 / \mu_{k}-1}\right) \\
& +\frac{K}{2 N} \log \left(1+\frac{2 N \operatorname{SNR}}{K^{2}} \sum_{r \in \mathcal{D}(k)} \frac{\left|\alpha_{r}\right|^{2}}{1 / \mu_{k}-1}\right),
\end{aligned}
$$

where SNR is the signal to noise ratio without considering any fading. The above mutual expression is a result of two mutual information. The first mutual information is between the user $k$ and the base station and the second mutual information is between the relays belonging to the decoding set, $\mathcal{D}(k)$ to the base station. The outage probability between the user $k$ and the base station over all possible decoding sets, $\mathcal{D}(k)$ is given by

$$
\operatorname{Pr}[I<R]=\sum_{\mathcal{D}(k)} \operatorname{Pr}[\mathcal{D}(k)] \operatorname{Pr}[I<R \mid \mathcal{D}(k)] .
$$

We know that the decoding set $\mathcal{D}(k)$ is the set of relays only which are able to decode the information they received from other users. Hence for a relay to be in the decoding set it has to satisfy the below condition:

$$
\left|\alpha_{r}\right|^{2} \geq \frac{2^{\left(\frac{2 N^{2} R}{K^{2}}\right)}-1}{2 N \mathrm{SNR} / K^{2}}
$$


Hence the probability that a relay, $r$ participates in the decoding set is given by

$$
\begin{aligned}
\operatorname{Pr}[r \in \mathcal{D}(k)] & =\operatorname{Pr}\left[\left|\alpha_{r}\right|^{2} \geq \frac{2^{\left(\frac{2 N^{2} R}{K^{2}}\right)}-1}{2 N \mathrm{SNR} / K^{2}}\right] \\
& =1-\left(1-\exp \left[-\lambda_{r} \frac{2^{\left(\frac{2 N^{2} R}{K^{2}}\right)}-1}{2 N \mathrm{SNR} / K^{2}}\right]\right) \\
& =1-\exp \left[-\lambda_{r} \frac{2^{\left(\frac{2 N^{2} R}{K^{2}}\right)}-1}{2 N \mathrm{SNR} / K^{2}}\right],
\end{aligned}
$$

where $\alpha_{r}$ is the flat fading Rayleigh coefficient with the variance $1 / \lambda_{r}$.

\subsubsection{Fully-loaded CDMA Uplink}

For the fully-loaded system we have $K=N$. The mutual information can be obtained just substituting the above condition for the fully-loaded systems. And here this system will now have $1 / 2$ degrees of freedom. The mutual information expression which is conditioned on the decoding set is obtained in a similar fashion as shown in [21] and is given as follows:

$$
\begin{aligned}
I_{\mathrm{f}-\mathrm{CDMA}}= & \frac{1}{2} \log \left(1+\frac{2 \mathrm{SNR}}{K} \frac{\left|\alpha_{k}\right|^{2}}{1 / \mu_{k}-1}\right) \\
& +\frac{1}{2} \log \left(1+\frac{2 \mathrm{SNR}}{K} \sum_{r \in \mathcal{D}(k)} \frac{\left|\alpha_{r}\right|^{2}}{1 / \mu_{k}-1}\right) .
\end{aligned}
$$

\subsubsection{Overloaded CDMA Uplink}

Here in a overloaded system, we have $K>N$. We can only generate $N$ linearly independent spreading waveforms and the rest of $K-N$ users will have to use the spreading codes which are the linear combinations of the first $N$. But each of user still uses all of the spreading waveforms and hence $1 / 2$ is the degrees of the freedom here. Note that we have assumed in the previous section that $m=k$ and all of the users are cooperating. Since we now have a linearly independent spreading waveforms it is not possible to distinguish between the self-transmitted message and a cooperated user's transmission. And to remove this confusion, each relay will delay its transmission while its cooperating. Though doing this is not optimal, it helps in maintaining a full rank $\boldsymbol{R}$ matrix. So the mutual information between the user $k$ and the base station conditioned on the decoding set for an overloaded 
system is given by

$$
\begin{aligned}
I_{\mathrm{o}-\mathrm{CDMA}}= & \frac{1}{2} \log \left(1+\frac{2 \mathrm{SNR}}{K} \frac{\left|\alpha_{k}\right|^{2}}{1 / \mu_{k}-1}\right) \\
& +\frac{1}{2} \log \left(1+\frac{2 \mathrm{SNR}}{K} \sum_{r \in \mathcal{D}(k)} \frac{\left|\alpha_{r}\right|^{2}}{1 / \mu_{k}-1}\right) .
\end{aligned}
$$

The above expression is same as the fully-loaded since they both have common degrees of freedom. The difference between both the systems is the value of the cross-correlation matrix $\mathbf{R}$ and also the spreading waveforms.

\subsection{Performance measures for a SIC Receivers}

In our proposed scheme we apply the interference cancellation to the output of the MMSE detector which will further reduce the residual interference in the received signal. After obtaining the scalar fading model as shown in 3.29 using the Gaussian approximation of the MMSE filter output, we find out the signal-to-interference-noise ratios (SINR) for every user which is given by equation (3.30). Based on these values of SINR, we rank the users. The user which ever has the highest SINR is canceled out. That user's corresponding elements in the cross correlation matrix $\boldsymbol{R}$ and also its channel coefficient from $\boldsymbol{A}$ are removed. Later the new values of the $\boldsymbol{R}$ are found and then the received signal model is modified in the (5). Now this is again passed through the instantaneous linear MMSE filter and the corresponding SINR's are calculated based on (21). This procedure is repeated until all of user's interferences are canceled out. For each cycle of this interference cancellation, the mutual information values are also parallely tracked for the corresponding system using the same equation as in (15), (19) and (20). And these values can all be average for all of the cycles of cancellation and then the final performance of the SIC receiver is calculated. 


\subsection{Results}

Figures 4.1, 4.2 and 4.3 show the information-outage probability for the underloaded CDMA, fully-loaded CDMA and overloaded CDMA for our proposed schemes. Figures 4.4 and 4.5 indicate the comparison of information-outage probabilities using SIC receivers for both the MMSE and the decorrelator detector using by [21]. Note that we have also plotted the Laneman's space-time coded protocol outage probability curve in all of the results and this would be used like a line of reference for all the graphs. However the Laneman's case [22] employees inter-user orthogonality, orthogonal space-time coding, symbol synchronization and an optimal decoder. Because of all the above assumptions there is no interference among the users across the subchannels. More over it should be considered as a fully-loaded scenario since it has $N=K=m$.

Also plotted on Figures 4.1, 4.2 and 4.3 are the decorrelator used in [21] outage probabilities for the high SNR approximation which a modified decorrelator detector. We have also performed a Monte-Carlo simulation on the decorrelator used in [21] to find out the outage probabilities with out a high-SNR approximation. There is a loss of spectral efficiency between the [21] and that of Laneman's case and this the because of using inter-user nonorthogonality which is a result of using non-orthogonal spreading codes and asynchronism. The decorrelator used in [21] also uses a suboptimal decorrelator for detection which doesn't to handle the problems of non-orthogonality and asynchronism effectively. We aim to close this gap and improve the performance of the system by employing the inter-user nonorthogonality and asynchronism which is a more practical scenario than the Laneman's.

Fig. 4.1 shows the outage probability for a under-loaded CDMA uplink which operates in a condition $K<N$ and also we take that all the users in the system are cooperating. We took the values as $N=4$ and $K=3$.For simulating the decorrelator case, we take the expression of the mutual information from [21] and also for the High-SNR approximation case we use 


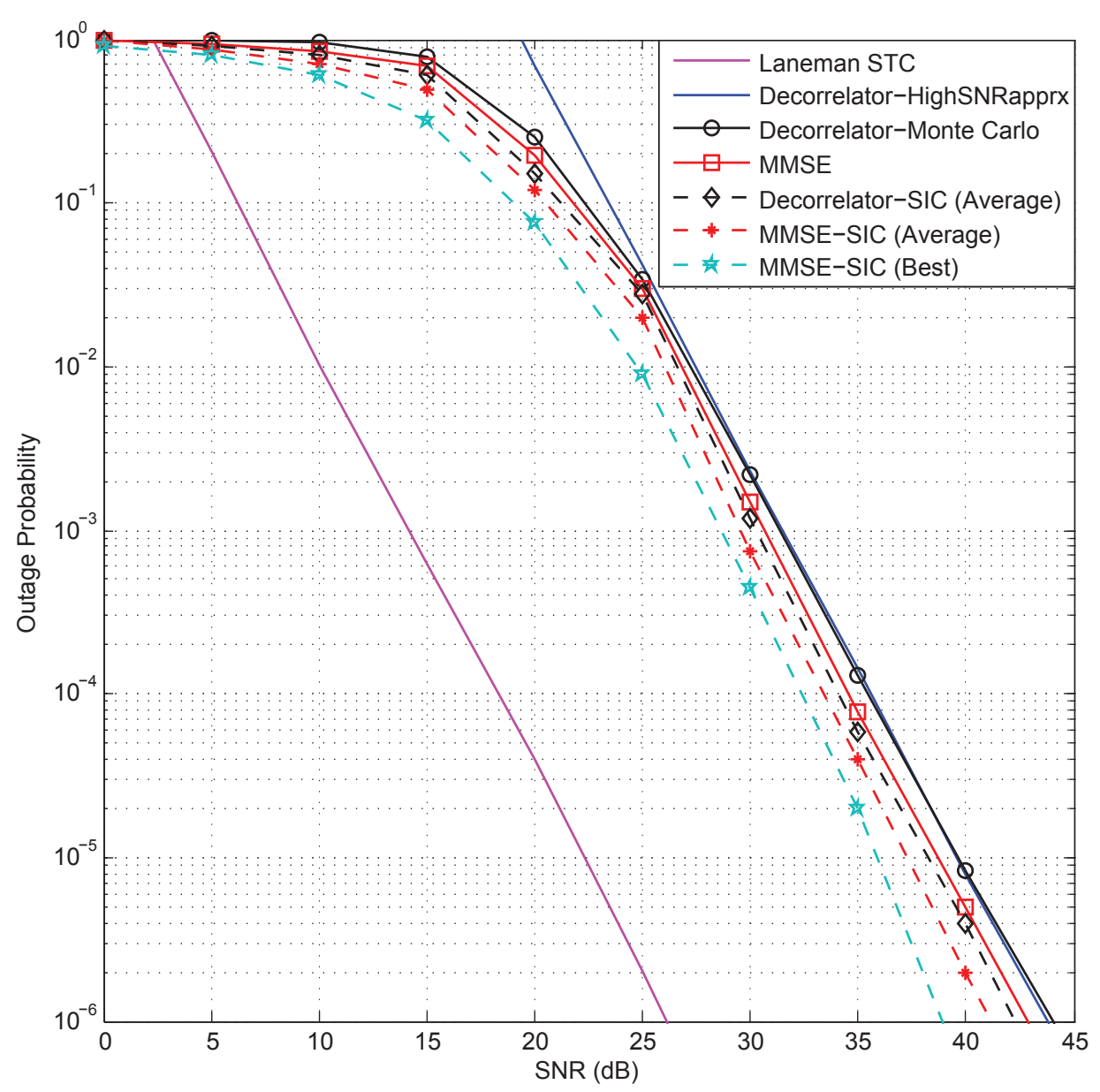

Figure 4.1: Information-outage probability performance of the proposed asynchronous underloaded CDMA uplink $(K<N)$ with $N=4, K=m=3$ without using high-SNR approximations. The spectral efficiency used as the threshold is $R=1 \mathrm{bit} / \mathrm{sec} / \mathrm{Hz}$. Also shown for comparison are the Laneman's synchronous STC $(m=4)$ and decorrelator outage probabilities with a high SNR approximation.

the following expression as seen in [21]:

$$
\begin{aligned}
P\left(I_{\mathrm{U}-\mathrm{CDMA}}<R \mid \boldsymbol{R}\right) \sim & {\left[\frac{2^{\left(\frac{2 N^{2} R}{K^{2}}\right)}-1}{2 N \mathrm{SNR} / K^{2}}\right]^{K} \times \sum_{D(k)} \lambda_{k, d}\left[\boldsymbol{R}^{-1}\right]_{k, k} } \\
& \times \prod_{r \in D(k)} \lambda_{r, d}\left[\boldsymbol{R}^{-1}\right]_{r, r} \\
& \times \prod_{r \notin D(k)} \lambda_{r, r}\left[\boldsymbol{R}^{-1}\right]_{r, r} \\
& \times A_{|D(k)|}\left(2^{\frac{2 N^{2} R}{K^{2}}}-1\right),
\end{aligned}
$$




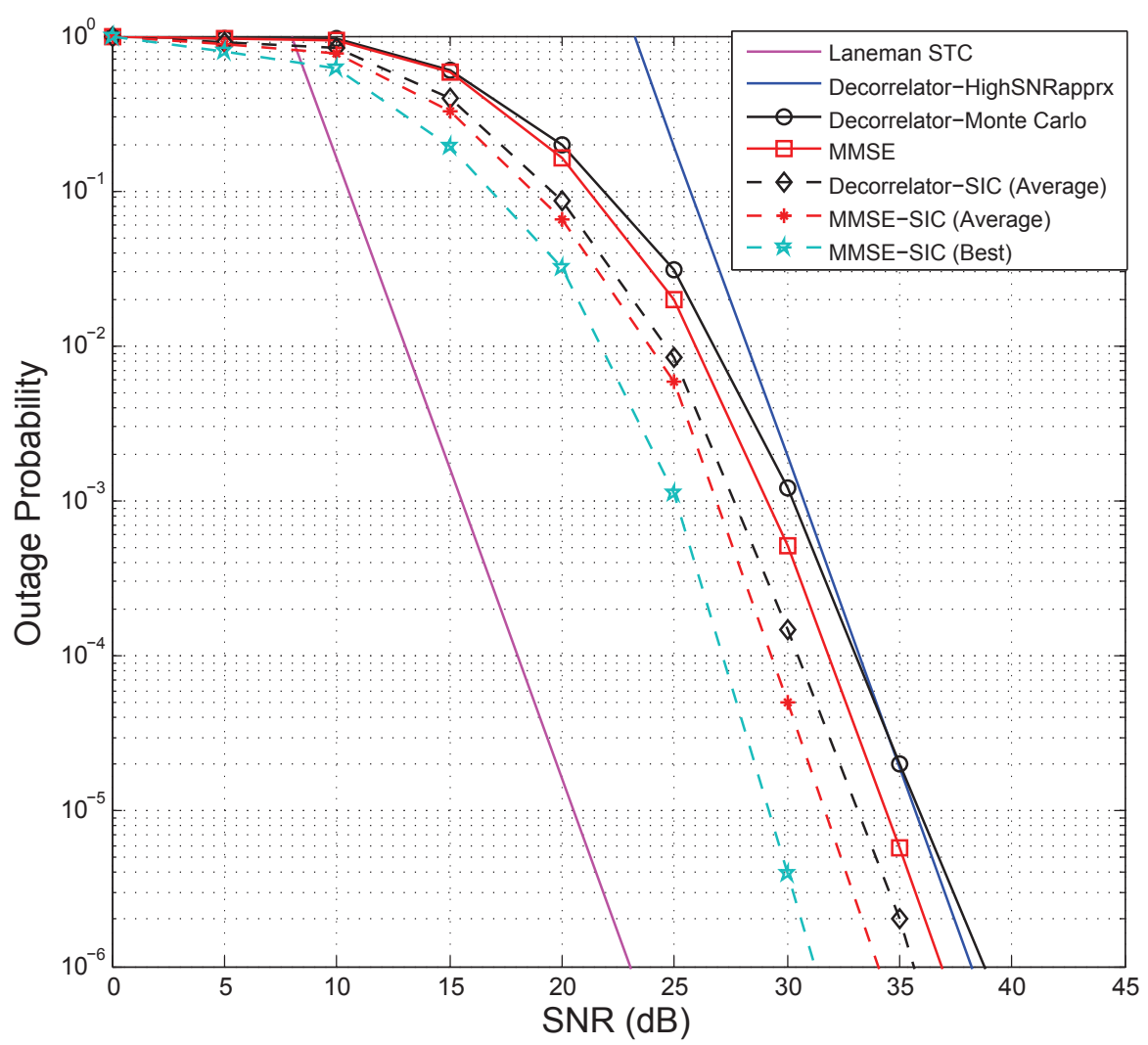

Figure 4.2: Information-outage probability performance of the proposed asynchronous fullyloaded CDMA uplink $(K=N)$ with $N=4, K=m=4$ without using high-SNR approximations. The spectral efficiency used as the threshold is $R=1 \mathrm{bit} / \mathrm{sec} / \mathrm{Hz}$. Also shown for comparison are the Laneman's synchronous STC $(m=4)$ and decorrelator outage probabilities with a high SNR approximation.

where the parameter $\lambda_{i, j}=1$. The above expression is only for the underloaded CDMA system. The expression is pretty similar for the fully-loaded CDMA and the overloaded CDMA with the change only in the degrees of freedom. For the Laneman's case we make sure that all the delays used are zeroed to achieve synchronism and we use the orthogonal spreading codes to achieve the inter-user orthogonality. It is seen that the use of SIC receivers has increased the performance. The use of only MMSE detectors does not seem to improve much since the decorrelator and MMSE perform almost the same but MMSE might show a slight improvement at high SNR's which is seen in the figure. The SIC receiver is used for both of the decorrelator[21] and the MMSE cases and curves plotted are the averages 


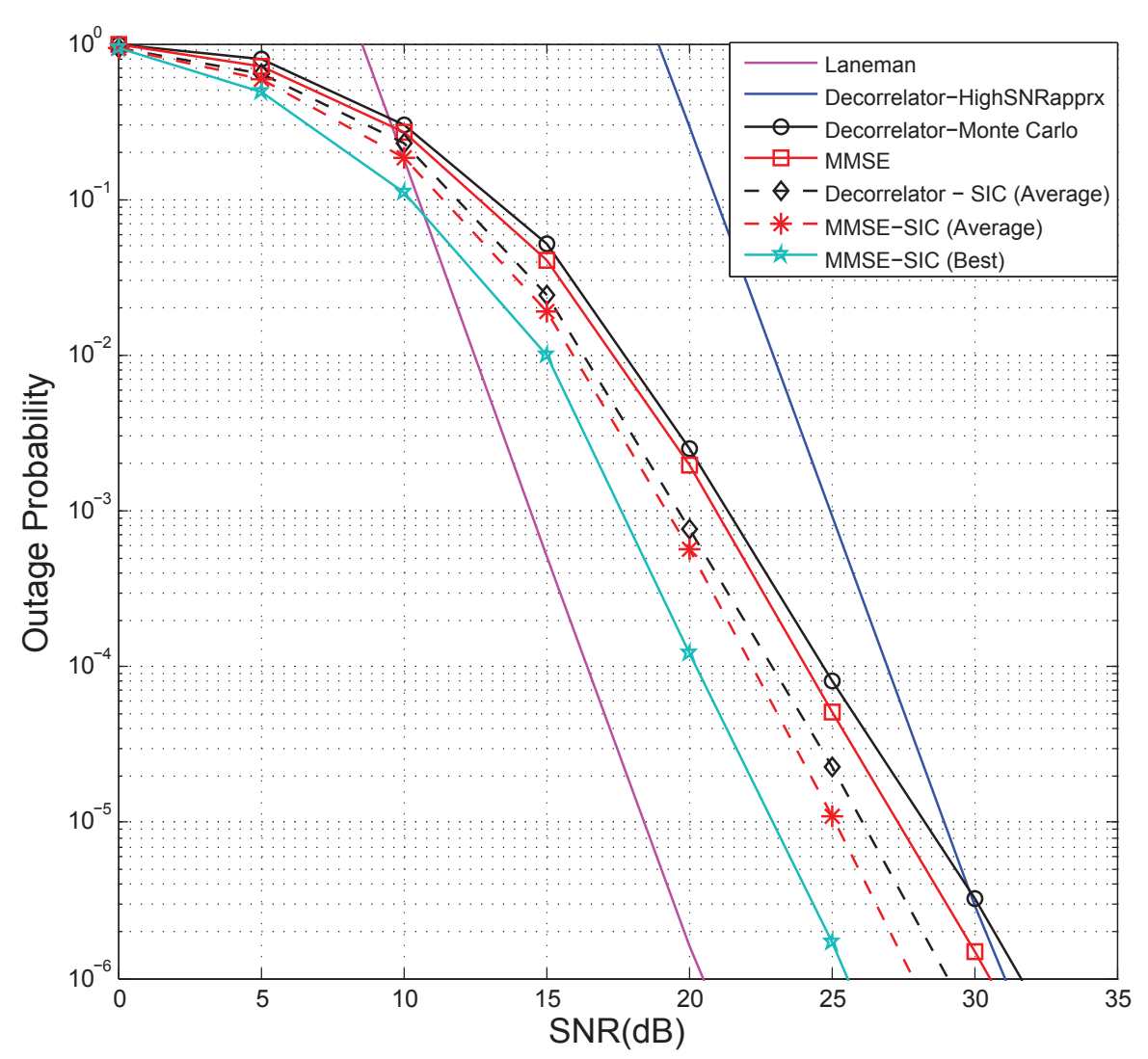

Figure 4.3: Information-outage probability performance of the proposed asynchronous overloaded CDMA uplink $(K>N)$ with $N=4, K=m=5$ without using high-SNR approximations. The spectral efficiency used as the threshold is $R=1 \mathrm{bit} / \mathrm{sec} / \mathrm{Hz}$. Also shown for comparison are the Laneman's synchronous STC $(m=4)$ and decorrelator outage probabilities with a high SNR approximation.

which are taken for all the stages of the interference cancellation. We note that if we make use of spreading codes and the synchronism between the symbols and this would make $\left[\boldsymbol{R}^{\mathrm{H}}+\sigma^{2} \boldsymbol{I}\right]^{-1}=\boldsymbol{I}$ in the expressions (15) and (19). And it would be then equivalent to the Laneman's space-time coded synchronous CDMA case.

Figure 4.2 indicates the information-outage probability curves for a fully-loaded CDMA uplink which is based on condition $K=N$. And here we consider the values of $K=N=4$. Similar to the previous case we have seen the same improvement here in the fully-loaded case. The SIC receiver here has performed well since there are more number of active users and this will also increase the number of cooperating users. Hence we have one extra stage of SIC cancellation which has increase the performance of the detectors. The improvement in the 
performance between the under-loaded and the fully-loaded is also because the underloaded doest seem to use all available degrees of freedom whereas fully-loaded utilizes all of them.

Figure 4.3 compares the outage probability curves for a overloaded CDMA uplink based on condition $K<N$ for our proposed asynchronous CDMA uplink. Here we consider the values of $N=4$ and $m=K=5$. The spreading codes considered in this case are not linearly independent, since we have only $N$ available spreading codes which have to be assigned to $K$ users. Hence we have to use some spreading codes which are the linear combinations of the first $N$ codes. This also helps to make sure we have a full rank cross correlation matrix $\boldsymbol{R}$. We notice the same bandwidth improvement compared to underloaded system as seen previously due to the use of full degrees of freedom. Similar to the other cases, the use of the post-interference cancellation on both the MMSE and decorrelator used in [21] has proved to reduce the SNR loss with respect to the Laneman's protocol.

The Figure 4.4 compares the information-outage probabilities of the decorrelator[21] with a post-interference cancellation. Shown in the plot are various stages of the cancellation involved and also the overall performance of the SIC case which is the average of all the stages. It is seen that the SNR loss was reducing gradually as we have increased the interference cancellations. For various stages of cancellation we obtain a new $\boldsymbol{R}$ by removing the user which has the highest interference and then proceed with that new $\boldsymbol{R}$ to the next stage. The curve shows the highest and the lowest achieved outage probability curves-highest being the last stage of cancellation which cancels out all of the cooperating users interference and removes out all their correlation from the $\boldsymbol{R}$ and the lowest is by the first stage which does not have any kind of interference cancellation and its $\boldsymbol{R}$ matrix has all the interferences included. similarly, Fig 4.5. indicates the same outage probabilities of the proposed MMSE detector with interference cancellation. We use the mutual informations shown from one of equations (4.1) and (4.5) for each stage of cancellation to find the corresponding outage probabilities. 


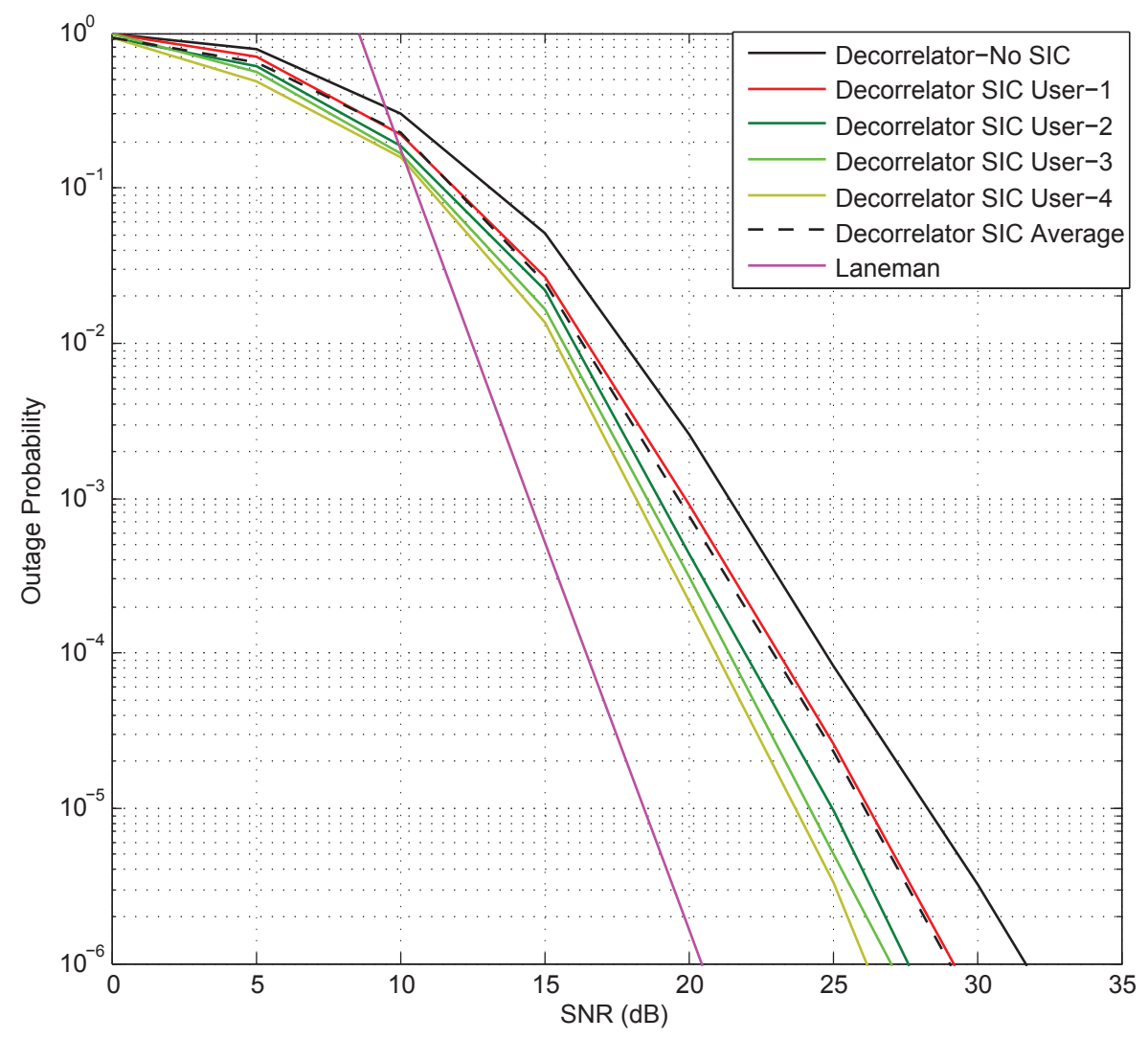

Figure 4.4: Comparison of Information-outage probability performance of the proposed asynchronous overloaded CDMA uplink $(K>N)$ with $N=4, K=m=5$ for different users of the decorrelator with SIC without using any SNR approximations. The spectral efficiency used as the threshold is $R=1 \mathrm{bit} / \mathrm{sec} / \mathrm{Hz}$. Also shown for comparison is the Laneman's synchronous STC $(m=4)$ 


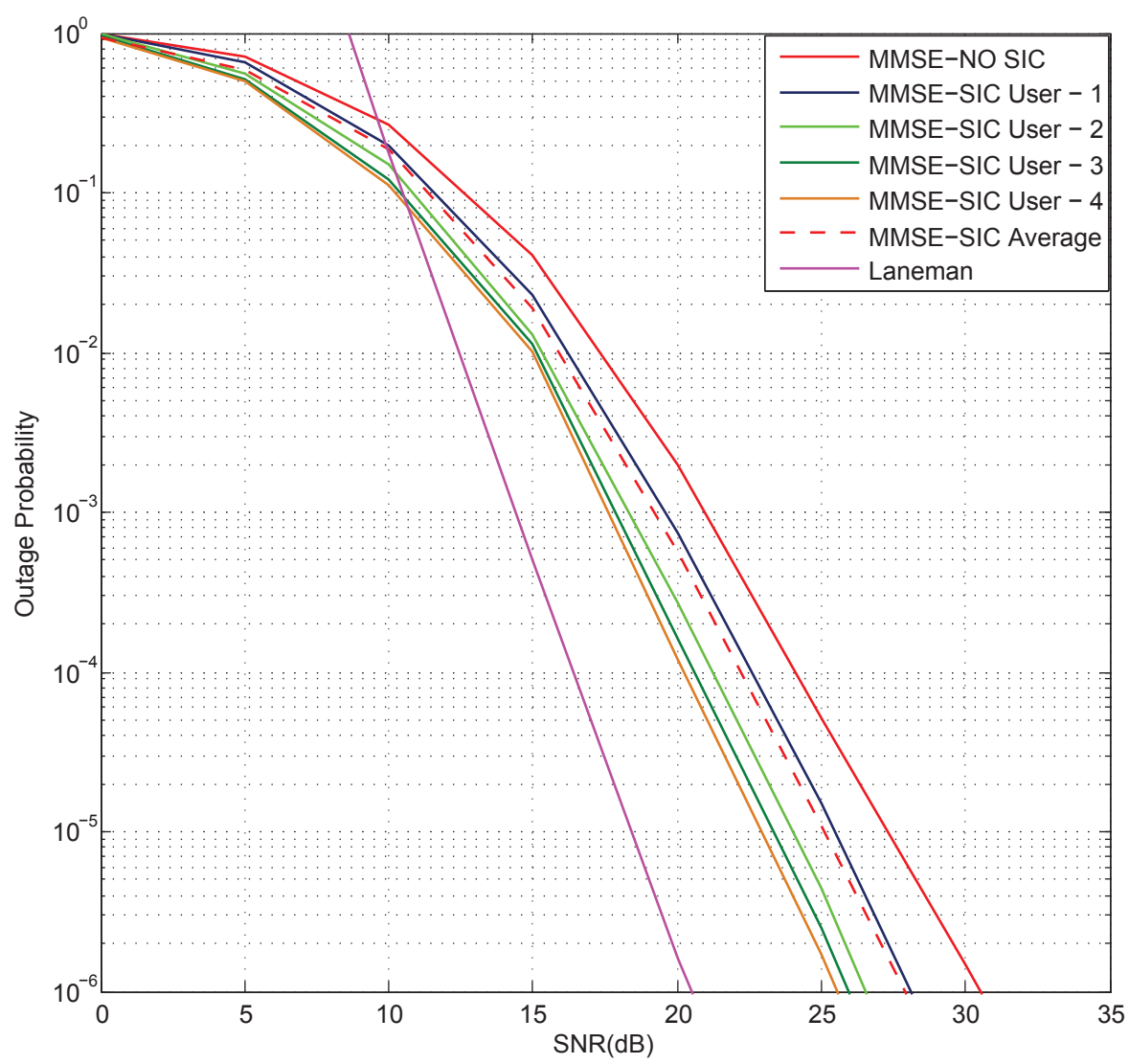

Figure 4.5: Comparison of Information-outage probability performance of the proposed asynchronous overloaded CDMA uplink $(K>N)$ with $N=4, K=m=5$ for different users of the MMSE detector with SIC without using any SNR approximations. The spectral efficiency used as the threshold is $R=1 \mathrm{bit} / \mathrm{sec} / \mathrm{Hz}$. Also shown for comparison is the Laneman's synchronous $\operatorname{STC}(m=4)$ 


\section{Chapter 5}

\section{Conclusion and Future Work}

In this paper, we have analyzed the performance of the proposed asynchronous CDMA uplink using the SIC receivers while maintaining the inter-user orthogonality and the asynchronism. The system consider here has full-duplex user and hence the users will be able to transmit and receive in the same frequency. The multiple access interference at the receiver is removed by using passing the received signals through a series of matched filter and these matched filter outputs are fed to a linear instantaneous MMSE filter. Then the interference cancelers are then used to further remove the residual interface from the MMSE filter output. We have used the information-outage probability as the performance measure to evaluate the performance of the above proposed scheme in the underloaded CDMA, fully-loaded CDMA and overloaded CDMA systems. We also have simulated the Laneman's space-time coded synchronous protocol and the [21]'s work where she implemented the same proposed scheme with a suboptimal decorrelator detector. The Laneman's case does not consider the nonorthogonality or the asynchronism and hence it does not have any of the problems associated with these two constraints. The decorrelator's case [21] on the other considers both of the constraints but due to the use of the highly suboptimal decorrelator detector in the receiver using which we have a large amount SNR loss in its performance. By using the proposed post-interference cancellation after the MMSE filter we were able to close this gap and have reduced the SNR losses we have seen [21]. We compared the interference cancellation for both the modified decorrelator design used by [21] and the proposed MMSE detector. It was seen that MMSE with interference cancellation worked better than that of the decorrelator. 
We have also compared how different stages of the interference cancellation performs and also have observed the highest achieved performance in those individual stages by plotting the outage probabilities at the end of each stage. By examining all the above results, we assert that the SIC receivers performs better than the others detectors previously used in other works and the SNR loss is not completely reduced as we have considered a design which is more feasible to use and more practical in nature than compared to the one used by Laneman[22]. 


\section{References}

[1] H. Palakurthi and D. Reynolds, "The Performance of Asynchronous Cooperative Diversity with SIC Receivers," 21 $1^{\text {st }}$ Virginia Tech Symposium on Wireless Personal Communications, June 2010.

[2] E. Dahlman, H. Ekstrom, A. Furuskar, Y. Jading, J. Karlsson, M. Lundevall, and S. Parkvall, "The 3G Long-Term Evolution - Radio Interface Concepts and Performance Evaluation," Vehicular Technology Conference, 2006. VTC 2006-Spring. IEEE 63rd, vol. 1, pp. 137-141, May 2006.

[3] Glisic, S. G., Advanced Wireless Communications: $4 G$ Technology, John Wiley and Sons, 2007.

[4] A. Ghosh, D.R. Wolter, J.G. Andrews, and R.; Chen, "Broadband wireless access with WiMax/802.16: current performance benchmarks and future potential," IEEE Commmunications Magazine, vol. 43, pp. 129-136, Feb 2005.

[5] E. Gregori G. Anastasi, M. Conti, "Ieee 802.11 ad hoc networks: protocols, performance and open issues," in Ad Hoc Networking, chapter 3. IEEE Press Wiley, New York, 2003.

[6] D. N. C. Tse, P. Viswanath, Fundamentals of Wireless Communication, Cambridge University Press, 2005.

[7] V. Tarokh, N. Seshadri, and A. R. Calderbank, "Space-time codes for high data rate wireless communications: Performance criterion and code construction.," IEEE Trans. Inform. Theory, vol. 44, no. 2, pp. 744-765, Mar. 1998.

[8] S. Alamouti, "A simple transmit diversity technique for wireless communications," IEEE J. Select. Areas Commun., vol. 16, no. 8, pp. 1451-1458, Oct. 1998.

[9] T. Kailath A. L. Paulraj, "Increasing capacity in wireless broadcast systems using distributed transmission/directional reception (dtdr)," 1994.

[10] İ. Telatar, "Capacity of multi-antenna gaussian channels," European Trans. Telecom., vol. 10, no. 6, pp. 585-595, Nov. 1999.

[11] G. Foschini and M. Gans, "On the Limits of wireless communications in a fading environment when using multiple antennas," Wireless Personal Commun, vol. 6, no. 3, pp. 311-335, 1998. 
[12] A. Paulraj, D. Gore, R. Nabar, H. Bölcskei, "An overview of MIMO communications - A key to gigabit wireless," Proceedings of the IEEE, vol. 92, no. 2, pp. 198-218, Feb. 2004.

[13] A. Goldsmith, Wireless Communications, Cambridge University Press., 2005.

[14] Y. Hua, Y. Mei and Y. Cheng, "Wireless antennas - making wireless communication perform like wireline communication," in IEEE Topical conference on Wireless Communication Technology, Honolulu, Hawaii, Oct. 2003, pp. 47-73.

[15] A. Sendonaris, E. Erkip, B. Aazang, "User cooperative diversity - Part I: System description," IEEE Trans. Communications, vol. 51, no. 1, pp. 1927-1938, Nov. 2003.

[16] N. Laneman and G. Wornell,, "Energy-Efficient Antenna Sharing and Relaying for Wireless Networks," in IEEE Wireless Comm. and Networking Conf. (WCNC), Chicago, IL, Sept. 2000, pp. 7-12.

[17] N. Laneman, D. N. C. Tse and G. Wornell,, "An Efficient Protocol for Realizing Cooperative Diversity in Wireless Networks," in IEEE Int. Symp. Information Theory (ISIT), Washington, DC, June 2001, p. 294.

[18] N. Laneman, D. Tse, and G. Wornell,, "Cooperative diversity in wireless networks: efficient protocols and outage behavior," IEEE Trans.Inform. Theory, vol. 50, no. 12, pp. 3062-3080, Dec. 2004.

[19] A. Scaglione and Y.Hong, "Opportunistic Large Arrays: Cooperative Transmission in Wireless Multihop Ad hoc networks to reach far distances," IEEE Trans. SP '03, vol. 51, no. 8, pp. 2082-2092, August 2003.

[20] S. Wei, D. L. Goeckel and M. C. , "Asynchronous cooperative diversity," IEEE Transactions on Wireless Communications, vol. 5, no. 6, pp. 1547-1557, June 2006.

[21] K. Vardhe, D. Reynolds, and M. C. Valenti, "The Performance of Multi-User Cooperative Diversity in an Asynchronous CDMA Uplink," IEEE Transactions on Wireless Communications, vol. 7, no. 5, pp. 1930-1940, May 2008.

[22] N. Laneman and G. Wornell, "Distributed space time coded protocols for exploiting cooperative diversity in wireless networks," IEEE Tran. Info. Theory, vol. 49, no. 10, pp. 2415-2425, Oct. 2003.

[23] J. Winters, "On the capacity of radio communication systems with diversity in a Rayleigh fading environment," IEEE Journal on Selected Areas in Communications, vol. 5, no. 5, pp. 871-878, June 1987.

[24] G. Foschini, "Layered space-time architecture for wireless communication in a fading environment when using multi-element antennas.," Bell Labs. Tech. Journal, vol. 1, no. 2, pp. 41-59, Autumn 1996. 
[25] G. J. Foschini, G. D. Golden, R. A. Valenzuela, and P. W. Wolniansky,, "Simplified processing for high spectral efficiency wireless communication employing multiple-element arrays," Wireless Personal Communications, vol. 6, pp. 311-335, March 1999.

[26] V. Tarokh, H. Jafarkhami, and A. R. Calderbank, "Space-time block codes from orthogonal designs," IEEE Trans. Inform. Theory, vol. 45, no. 5, pp. 1456-1467, July 1999.

[27] L. Zheng, D. Tse, "Diversity and multiplexing: A fundamental tradeoff in multiple antenna channels," IEEE Transactions on Inform. Theory, vol. 49, pp. 1073-1096, May 2003.

[28] G. J. Foschini, G. D. Golden, R. A. Valenzuela, and P. W. Wolniansky,, "Detection Algorithm and Initial Laboratory Results using the V-BLAST Space-Time Communications Architecture," Electronics Letters, vol. 35, no. 7, pp. 14-15, Jan. 1999.

[29] A. Nosratinia, T. Hunter, and A. Hedayat,, "Cooperative communication in wireless networks," IEEE Commun. Magazine, vol. 42, no. 10, pp. 74-80, Oct. 2004.

[30] B. Sklar, Digital Communications - Fundamentals and Applications, Pearson Education, 2001.

[31] S. Verdú, Multiuser Detection, Cambridge, UK: Cambridge University Press, 1998.

[32] S. Verdú, "Minimum probability of error for asynchronous gaussian multiple-access channels," IEEE Trans. Inform. Theory, vol. 32, pp. 85-96, Jan. 1986.

[33] R. Lupas and S. Verdú, "Linear multiuser detectors for synchronous code-division multiple-access channels," IEEE Trans. Inform. Theory, vol. 35, no. 1, pp. 123-136, Jan. 1989.

[34] Z. Xie, R.Short, and C. K. Rushforth, "A family of suboptimum detectors for coherent multiuser communications," IEEE J. Select. Areas Commun., vol. 8, pp. 683-690, May. 1990.

[35] A. J. Viterbi, "Very low rate convolutional codes for maximum theoretical performance of spread-spectrum multiple-access channels," IEEE J. Select. Areas Commun., vol. 8, no. 4, pp. 641-649, May 1990.

[36] P. Patel, and J. Holtzman, "Analysis of a simple successive interference cancellation scheme in a DS/CDMA system," IEEE J. Select. Areas Commun., vol. 12, no. 5, pp. 796-807, June 1994.

[37] M. K. Varanasi and B. Aazhang, "Multistage detection in asynchronous code-division multiple-access communications," IEEE Trans. Commun., vol. 38, no. 4, pp. 509-519, Apr. 1990.

[38] X. Wang, and H. V. Poor, "Blind multiuser detection: A subspace approach," IEEE Trans. Inform. Theory, vol. 44, no. 2, pp. 677-690, Mar. 1998. 
[39] L. H. Ozarow, S. Shamai (Shitz), and A. D. Wyner, "Information theoretic considerations for cellular mobile radio," IEEE Trans. Veh. Technol., vol. 43, no. 5, pp. 359378, May 1994.

[40] H. V. Poor and S. Verdú, "Probability of error in MMSE multiuser detection," IEEE Trans. Inform. Theory, vol. IT-43, pp. 858-871, May 1997.

[41] X. Wang and H. V. Poor, "Iterative (Turbo) soft interference cancellation and decoding for coded CDMA," IEEE Transactions on Communications, vol. 46, no. 7, pp. 10461061, July 1999. 